\title{
An Integrated Approach to Workflow Mapping and Task Scheduling for Delay Minimization in Distributed Environments
}

\author{
Daqing Yun ${ }^{\mathrm{a}}$, Chase Qishi Wu ${ }^{\mathrm{a}, *}$, Yi Gu ${ }^{\mathrm{b}}$ \\ ${ }^{a}$ Department of Computer Science, New Jersey Institute of Technology, Newark, NJ 07102 \\ ${ }^{b}$ Department of Computer Science, Middle Tennessee State University, Murfreesboro, TN 37132
}

\begin{abstract}
Many scientific applications feature large-scale workflows consisting of computing modules that must be strategically deployed and executed in distributed environments. The endto-end performance of such scientific workflows depends on both the mapping scheme that determines module assignment, and the scheduling policy that determines resource allocation if multiple modules are mapped to the same node. These two aspects of workflow optimization are traditionally treated as two separated topics, and the interactions between them have not been fully explored by any existing efforts. As the scale of scientific workflows and the complexity of network environments rapidly increase, each individual aspect of performance optimization alone can only meet with limited success. We conduct an in-depth investigation into workflow execution dynamics in distributed environments and formulate a generic problem that considers both workflow mapping and task scheduling to minimize the end-to-end delay of workflows. We propose an integrated solution, referred to as Mapping and Scheduling Interaction (MSI), to improve the workflow performance. The efficacy of MSI is illustrated by both extensive simulations and proof-of-concept experiments using real-life scientific workflows for climate modeling on a PC cluster.
\end{abstract}

Keywords: Scientific workflows, workflow mapping, on-node scheduling, end-to-end delay.

\section{Introduction}

The processing and analysis of simulation or experimental datasets generated in nextgeneration e-Science require the construction and execution of domain-specific workflows in distributed network environments, such as clusters, grids, or clouds for collaborative research and discovery $[1,2]$. Such scientific workflows typically consist of many interdependent

\footnotetext{
${ }^{*}$ Corresponding author

Email addresses: daqing.yun@njit.edu (Daqing Yun), chase.wu@njit.edu (Chase Qishi Wu), yi.gu@mtsu.edu (Yi Gu)
}

Preprint submitted to Journal of Parallel and Distributed Computing

June 18, 2015

(C) 2015. This manuscript version is made available under the Elsevier user license http://www.elsevier.com/open-access/userlicense/1.0/ 
computing modules ${ }^{1}$, and are managed and executed by either special- or general-purpose workflow engines such as HTCondor/DAGMan [3-5], Kepler [6], Pegasus [7, 8], Triana [9], Askalon [10] and Sedna [11]. In general, a workflow system first assigns component modules to a set of networked nodes (i.e. mapping ${ }^{2}$ ) in the deployment phase and then decides the order or priority of module execution (i.e. scheduling) at runtime.

The workflow mapping problem is well known to be NP-complete and non-approximable for planar and bipartite workflow graphs [12], and has been extensively investigated in the literature to minimize the End-to-end Delay (ED) or makespan of a workflow. No matter which mapping scheme is applied, it is often inevitable to assign multiple modules to the same node (i.e. node reuse) for better utilization of limited computing resources, leading to possible concurrent module execution. In such cases, the node's computing resources are generally allocated by kernel-level CPU scheduling policies, such as the round-robin algorithm to ensure fine-grained fair-share (FS). Similarly, a network link's bandwidth is also fairly shared by multiple independent data transfers that take place concurrently over the same link through the use of the widely deployed TCP or TCP-friendly transport methods. Such system-inherent fair-share scheduling mechanisms could reduce the development and implementation efforts of workflow systems, but may not always yield the best workflow performance, especially in distributed environments. The scheduling effect was considered in some recent efforts including [13], where a Critical Path ${ }^{3}$-based Priority Scheduling (CPPS) algorithm is proposed to improve the end-to-end performance of a workflow under a given mapping scheme that is initially calculated based on FS scheduling.

As well recognized, the performance of scientific workflows depends on both the mapping scheme and the on-node scheduling policy, which are traditionally treated in two separate realms. As the scale of scientific workflows and the complexity of network environments rapidly increase, each individual aspect of performance optimization alone can only meet with limited success. In fact, there exists a certain level of interactions between mapping and scheduling, which could be exploited to further improve the end-to-end workflow performance. We shall use a simplified numerical example on a workflow consisting of 6 modules as shown in Fig. 1 to illustrate how mapping and scheduling can interact with each other, leading to different end-to-end performances. For simplicity, we ignore the computational

\footnotetext{
${ }^{1}$ Workflow modules are also referred to as tasks/subtasks, activities, stages, jobs, or transformations in different contexts.

${ }^{2}$ Workflow mapping is occasionally referred to as a scheduling problem in the literature. In this paper, we use the term "mapping" to differentiate it from the workflow on-node scheduling problem under study within the same framework.

${ }^{3}$ The critical path in the context of workflow optimization refers to the longest path of execution time.
} 


\begin{tabular}{|ccc|}
\hline Module & CR & Mapped To (PP) \\
\hline$W_{0}$ & 0 & $v_{0}(---)$ \\
$W_{1}$ & 40 & $V_{1}(10)$ \\
$W_{2}$ & 10 & $V_{1}(10)$ \\
$W_{3}$ & 20 & $V_{2}(10)$ \\
$W_{4}$ & 10 & $V_{2}(10)$ \\
$W_{5}$ & 0 & $V_{3}(---)$ \\
\hline \multicolumn{3}{c}{ Workflow parameters }
\end{tabular}

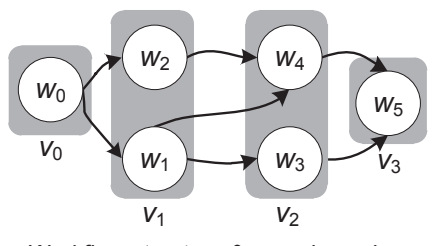

Figure 1: A simple example illustrating the interactions between mapping and scheduling.
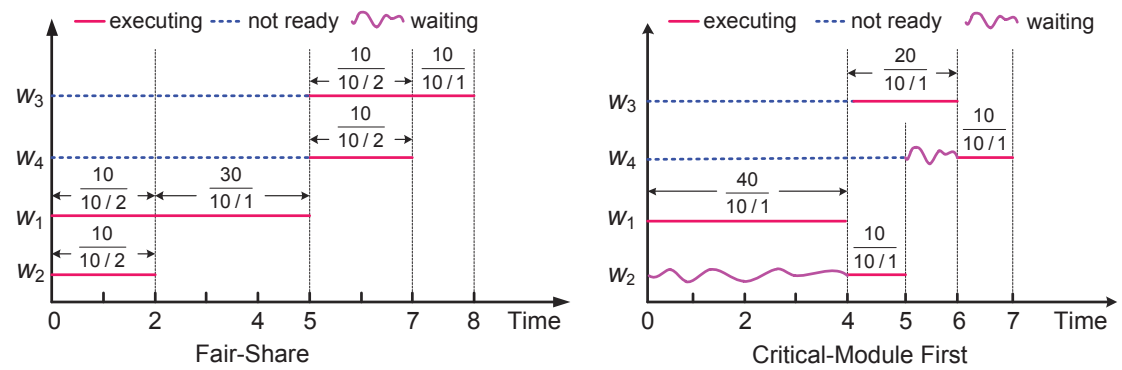

Figure 2: Module execution dynamics based on: fair-share vs. critical-module-first.

requirement $(\mathrm{CR})$ of the start/end module (i.e. $w_{0}$ and $w_{5}$ ). The initial mapping scheme maps modules $w_{1}$ and $w_{2}$ to node $v_{1}$, and maps modules $w_{3}$ and $w_{4}$ to node $v_{2}$. All the nodes have identical processing power (PP) of 10 units/s.

Under the given mapping scheme in Fig. 1, the execution dynamics of the entire workflow are shown in Fig. 2 based on FS scheduling. Along the $x$-axis, starting from time point 0 , it takes 2 seconds of fair share between $w_{1}$ and $w_{2}$ on $v_{1}$ to finish $w_{2}$ and then another 3 seconds of exclusive execution of $w_{1}$ to finish $w_{1}$ at time point 5 . At this point, $w_{3}$ and $w_{4}$ become "ready". It takes 2 seconds of fair share between $w_{3}$ and $w_{4}$ on $v_{2}$ to finish $w_{4}$ and another 1 second of exclusive execution of $w_{3}$ to finish $w_{3}$ at time point 8 . Therefore, the end-to-end delay based on FS scheduling is 8 seconds on the critical path: $w_{0} \rightarrow w_{1} \rightarrow w_{3} \rightarrow w_{5}$. Based on this FS schedule, by employing a naive on-node scheduling policy that always executes the critical module exclusively first in case of resource competition, we can cut down the end-to-end delay from 8 seconds to 7 seconds as shown in Fig. 2. However, this new schedule leads to a different critical path, i.e. $w_{0} \rightarrow w_{2} \rightarrow w_{4} \rightarrow w_{5}$. Such a shift of the critical path indicates that the original mapping scheme computed by any critical path-based mapping algorithm based on FS scheduling may no longer be the optimal one, hence requiring reexamining the mapping procedure.

Modern systems and networks provide various mechanisms to perform resource scheduling that goes beyond the system-inherent FS scheduling. For example, the allocation of 
system resources (mainly CPU cycles) on a host among concurrent modules or jobs could be controlled by assigning different CPU quantum values through "nice" or "renice" commands or some specialized tools such as "CGroups" [14] in Linux. Meanwhile, apart from TCP-based data transfer over default best-effort IP paths, the allocation of network resources (mainly bandwidths) could also be controlled on dedicated channels, as exemplified by high-performance networks with the capability of bandwidth reservation $[15,16]$ and the Internet using QoS technologies such as DiffServ [17], IntServ [18], RSVP [19], and MPLS [20] to implement fine-grained bandwidth control. Therefore, it is practically feasible to perform more sophisticated control for module execution and data transfer than the default FS scheduling to achieve a higher level of workflow performance.

In this paper, we conduct an in-depth investigation into workflow execution dynamics and formulate a generic workflow optimization problem that considers both workflow mapping and scheduling. We propose an integrated solution, referred to as Mapping and Scheduling Interaction (MSI), to optimize workflow performance. MSI takes an iterative approach to perform the mapping-scheduling cycle in order to minimize the end-to-end delay of a distributed workflow. The performance superiority of the proposed solution is illustrated by extensive simulations and proof-of-concept experiments using domain-specific scientific workflows in real network environments.

The rest of this paper is organized as follows. Section 2 conducts a survey of related work. Section 3 formulates the workflow optimization problem. Section 4 details the design of MSI algorithm. Section 5 evaluates the algorithm performance through simulations and experiments.

\section{Related Work}

We conduct a background survey on workflow optimization, which has been the focus of research in recent years $[21,13,22,23]$. Generally, there are two aspects of optimizing distributed tasks to improve scientific workflow performance: i) assigning the component modules in a workflow to suitable computing resources, referred to as workflow mapping; and ii) deciding the execution order/priority and resource sharing policy among concurrent modules on computer nodes or processors, referred to as workflow on-node scheduling. Both aspects have been extensively studied in various contexts due to their theoretical significance and practical importance [24-33].

The existing workflow mapping algorithms can be roughly classified into the following categories: i) Graph-based methods [34, 35], which tackle the mapping problem using graph theory. The subgraph isomorphism has been proven to be NP-complete and the complexity 
of graph isomorphism is still unknown; ii) List scheduling approaches [36, 37], most of which employ a critical path-based procedure; iii) Clustering algorithms [38, 39], which assume an unlimited number of processors and thus are not feasible in reality; iv) Duplication-based algorithms [40,30], most of which have a complexity of $O\left(n^{4}\right)$ or higher for $n$ nodes; v) Guided random search such as genetic algorithms [41, 42], which often require additional efforts to determine an appropriate termination condition and usually does not have performance guarantee.

In early years when networked resources were still scarce, workflow modules were typically mapped to tightly coupled homogeneous systems such as multiprocessors $[12,43,39$, 37]. As distributed platforms such as clusters, grids, and clouds are rapidly developed and deployed, research efforts have shifted to workflow mapping in heterogeneous environments to utilize distributed resources at different geographical locations across wide-area networks, which facilitate a much larger scope of collaborations among different institutes [30, 38, 44]. However, some of the aforementioned efforts assume that networks are fully connected [28] or only consider independent tasks in the workflow [32] without any resource sharing. These assumptions are valid under certain circumstances, but may not be always practical nowadays and thus are difficult to be incorporated in real-life workflow applications.

Considering the limit in both the scope and the amount of networked resources, multiple modules are often mapped to the same node, which necessitates the scheduling of concurrent modules. Task scheduling has been a traditional topic in computer science, and fair-share scheduling is widely adopted in real systems. Some traditional scheduling algorithms consider multiple independent processes running on a single processor with a goal to optimize the waiting time, response time, and turnaround time of individual processes, or the throughput of the entire system. Garey et al. compiled a great collection of similar or related multi-processor scheduling problems in [45], most of which are NP-complete and were tackled by various heuristics such as list scheduling and simple level algorithm [46]. However, the research on module scheduling specifically tailored to scientific workflows in distributed environments is still quite limited. Many existing efforts on workflow scheduling assume non-preemption of module execution [33, 32], which oversimplifies the execution dynamics in resource sharing and may not sufficiently model the complexity of real-life applications in wide-area networks.

The work in [13] investigates the task scheduling problem for Minimum End-to-end Delay (MED) in unitary processing applications under a given workflow mapping scheme, and shows that the performance could be improved by deciding an appropriate resource sharing policy among concurrent workflow modules mapped to the same node. In this paper, we go 
beyond the traditional mapping and scheduling approaches by integrating both to achieve a higher level of workflow performance.

\section{Problem Formulation}

In this section, we construct analytical cost models and formally define a generic workflow optimization problem that takes both mapping and scheduling into consideration.

\subsection{Cost Models}

We model a scientific workflow as a Directed Acyclic Graph (DAG) $G_{w}\left(V_{w}, E_{w}\right),\left|V_{w}\right|=$ $m$, where vertices represent computing modules starting from the start module $w_{0}$ to the end module $w_{m-1}$. A directed edge $e(i, j) \in E_{w}$ in the DAG represents the dependency between a pair of adjacent modules $w_{i}$ and $w_{j}$. As shown in Fig. 3(a), module $w_{i}$ receives a data input of size $z(\cdot, i)$ from each of its preceding modules $w_{k}, \ldots, w_{j}$ and performs a predefined computational routine. Generally, the computational requirement (CR) or workload of a module depends on the module's computational complexity and input data size: the former is decided by the specific algorithm implemented in the module and the latter defines the problem size or the amount of data to be processed. We model the CR of a module $m_{i}$ as a generic function $f_{i}$ of the aggregated input data $Z_{i}$ :

$$
C R_{i}=f_{i}\left(Z_{i}\right)=f_{i}(\underset{x=k}{\stackrel{j}{A}}(z(x, i))),
$$

where $Z_{i}$ is an aggregation of all input data sizes based on a certain aggregation function $A$. To make the computational complexity function generic, we do not specify the data aggregation method in our model, but make it concrete in practice. For example, in our simulations, we simply perform a summation operation on all the input data sizes for aggregation, i.e. $A=\sum$.

In this workflow model, we consider a module as an atomic execution unit and ignore inter-module communication cost on the same node. For a workflow with multiple start/end modules, we can always convert it to this model by adding a virtual start/end module with zero computational requirement and connecting it to all the original start/end modules with zero data transfer.

We model an overlay computer network as an arbitrary weighted graph $G_{c}\left(V_{c}, E_{c}\right)$, consisting of $\left|V_{c}\right|=n$ nodes interconnected by $\left|E_{c}\right|$ overlay links. The estimation of a computer node's processing power (PP) is a complex task since it involves a variety of host factors. For simplicity, in Fig. 3(b), we use a normalized variable $P P_{i}$ to represent the 


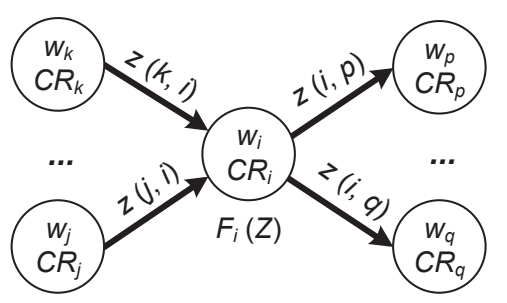

(a)

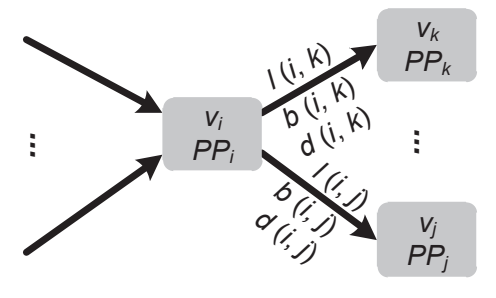

(b)

Figure 3: Cost models for computing modules, network nodes, and communication links: (a) scientific workflow, (b) computer network.

overall processing power of node $v_{i}$. The communication link $l(i, j)$ from $v_{i}$ to $v_{j}$ is associated with a certain bandwidth $b(i, j)$ and minimum link delay (MLD) $d(i, j)$. Generally, the start module $w_{0}$ serves as a data source on the source node $v_{0}$ without any computation to supply all initial data needed by the application and the end module $w_{m-1}$ performs a terminal task on the destination node $v_{n-1}$ without any further data transfer. This network model is generic to accommodate various distributed environments such as clouds, grids, and PC clusters.

The module's complexity together with the input data size decides the amount of CPU cycles needed to finish the execution of the computational routine defined in the module. The actual execution time of a module also depends on the capacity of the processing power of the mapped computer node as well as its availability at runtime. Our model estimates the execution time of workflow module $w_{i}$ on computer node $v_{j}$ as follows:

$$
T_{\text {exec }}\left(w_{i}, v_{j}\right)=\frac{C R_{i}}{P P_{j}}=\frac{f_{i}\left(Z_{i}\right)}{P P_{j}} .
$$

The transfer time of a large chunk of data over a communication link is mainly constrained by the link's bandwidth while the MLD can be a significant overhead for the transfer of a small amount of data whose size is comparable to the Maximum Transmission Unit (MTU) of the underlying network infrastructure. We estimate the transfer time of a message of data size $z$ over a communication link $l(i, j)$ as follows:

$$
T_{\text {trans }}(z, l(i, j))=\frac{z}{b(i, j)}+d(i, j) .
$$

Based on the above models, we may use an existing workflow mapping algorithm to compute a mapping scheme under the following constraints on workflow mapping and execution/transfer precedence [13]:

- Each module/edge is required to be mapped to only one node/link. 
Table 1: Notations in the cost models

\begin{tabular}{|l|l|}
\hline Notations & Definitions \\
\hline$G_{w}\left(V_{w}, E_{w}\right)$ & A workflow, $\left|V_{w}\right|=m$ \\
\hline$w_{i}$ & The $i^{t h}$ module in $G_{w}$ \\
\hline$t_{w_{i}}^{s}$ & The start time of module $w_{i}$ \\
\hline$t_{w_{i}}^{f}$ & The finish time of module $w_{i}$ \\
\hline$e(i, j)$ & The edge from module $w_{i}$ to $w_{j}$ \\
\hline$z(i, j)$ & The data size sent from $w_{i}$ to $w_{j}$ \\
\hline$C R_{i}$ & The workload of module $w_{i}$ \\
\hline$G_{c}\left(V_{c}, E_{c}\right)$ & A overlay network, $\left|V_{c}\right|=n$ \\
\hline$v_{i}$ & The $i^{t h}$ computer node \\
\hline$P P_{i}$ & The processing power of node $v_{i}$ \\
\hline$l(i, j)$ & The link from node $v_{i}$ to $v_{j}$ \\
\hline$b(i, j)$ & The bandwidth of link $l(i, j)$ \\
\hline$d(i, j)$ & The minimum link delay of link $l(i, j)$ \\
\hline$T_{t r a n s}(z, l(i, j))$ & The transfer time of data size $z$ over link $l(i, j)$ \\
\hline$T_{e x e c}(w, v)$ & The execution time of module $w$ on node $v$ \\
\hline$M_{G_{w} \rightarrow G_{c}}$ & An mapping scheme that maps $G_{w}$ to $G_{c}$ \\
\hline$M_{i}$ & The mapping scheme in the $i^{t h}$ round interaction of Alg. 1 \\
\hline$S_{\left\{w_{1}, w_{2}, \cdots, w_{k}\right\} \rightarrow v}$ & An on-node scheduling policy for $\left\{w_{1}, w_{2}, \cdots, w_{k}\right\}$ over node $v$ \\
\hline$S_{G_{w} \rightarrow G_{c}}$ & An entire on-node scheduling policy for $G_{w}$ on $G_{c}$ \\
\hline$S_{i}$ & The $i^{t h}$ interaction on-node scheduling policy in Alg. 1 \\
\hline$T_{i}$ & The execution time components of $G_{w}$ in the $i^{t h}$ round in Alg. 1 \\
\hline$T_{\left\langle G_{w}, G_{c}, M, S\right\rangle}$ & $\begin{array}{l}\text { The execution time components of } G_{w} \text { when mapped to } G_{c} \text { by } M_{G_{w}} \rightarrow G_{c} \\
\text { scheduled by } S_{G_{w} \rightarrow G_{c}}\end{array}$ \\
\hline$C P(T)$ & The critical path calculated based on time components $T$ \\
\hline$E D\left\langle G_{w}, G_{c}, M, S\right\rangle$ & $\begin{array}{l}\text { End-to-end delay of } G_{w} \text { when mapped to } G_{c} \text { by } M_{G_{w}} \rightarrow G_{c} \text { and scheduled by } \\
S_{G_{w} \rightarrow G_{c}}\end{array}$ \\
\hline
\end{tabular}

- A computing module cannot start execution until all its required input data arrives.

- A dependency edge cannot start data transfer until its preceding module finishes.

We define a workflow mapping scheme $M_{G_{w} \rightarrow G_{c}}$ that maps $G_{w}$ to $G_{c}$ as follows:

\section{Definition 1. Workflow Mapping Scheme}

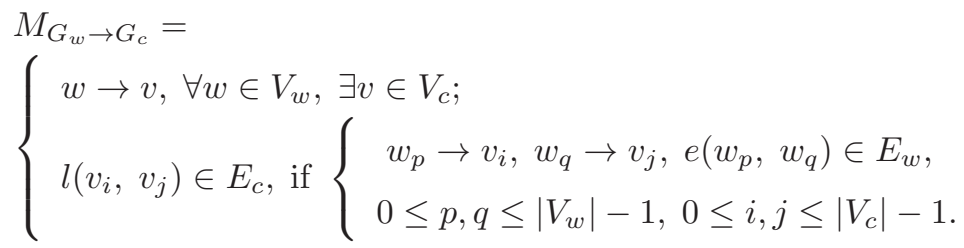

The requirements for a workflow mapping scheme defined in Def. 1 are two-fold: i) for any module $w$ in the workflow $G_{w}$, there must be a node $v$ in the computer network $G_{c}$, to which $w$ is mapped $(w \rightarrow v)$; ii) if modules $w_{p}$ and $w_{q}$ are mapped to nodes $v_{i}$ (i.e. $\left.w_{p} \rightarrow v_{i}\right)$ and $v_{j}$ (i.e. $\left.w_{q} \rightarrow v_{j}\right)$, respectively, and there is an edge $e\left(w_{p}, w_{q}\right)$ between $w_{p}$ and $w_{q}$ in the workflow $G_{w}$, there must be a link $l\left(v_{i}, v_{j}\right)$ between $v_{i}$ and $v_{j}$ in the network $G_{c}$.

We further convert the above workflow and network models to virtual graphs as follows: each dependency edge in the workflow is replaced with a virtual module whose computational requirement is equal to the corresponding data size, and each mapped network link 
is replaced by a virtual node whose processing power is equal to the corresponding bandwidth. This conversion facilitates workflow scheduling as we only need to focus on the module execution time.

When there are multiple modules (either actual workflow module or converted virtual module) mapped to one single computer node, the CPU time (quantum) allocated to each competing module is controlled by the kernel-level CPU scheduling policy, and the link bandwidth allocated to each data transfer is determined by the specific transport methods used on the end hosts. In the previous work [47-49,36], both CPU time and link bandwidth are assumed to be fairly shared through the use of a round-robin scheduling algorithm and a TCP or TCP-friendly transport protocol. In this work, we go beyond this assumption and use an On-node Scheduling Policy to determine resource sharing in a more fine-grained manner.

\section{Definition 2. On-node Scheduling Policy}

If a subset of modules $\left\{w_{1}, w_{2}, \cdots, w_{k}\right\}$ in a workflow $G_{w}$ are mapped to a computer node $v$ and there exists resource competition among these modules, an On-node Scheduling Policy for $\left\{w_{1}, w_{2}, \cdots, w_{k}\right\}$ on $v$, denoted as $S_{\left\{w_{1}, w_{2}, \cdots, w_{k}\right\} \rightarrow v}$, is a vector in which each element $s_{i}$ $(1 \leq i \leq k)$ is the resource share quantum for $w_{i}$ in a scheduling round.

Since an on-node scheduling policy is node-specific according to Def. 2, we use $S_{G_{w} \rightarrow G_{c}}$ to denote a full set of on-node scheduling policies for the entire workflow $G_{w}$ over computer network $G_{c}$. Once $M_{G_{w} \rightarrow G_{c}}$ and $S_{G_{w} \rightarrow G_{c}}$ are both determined for all modules and nodes, we could calculate the execution time of every workflow module and the End-to-end Delay (ED) of the entire workflow, as defined below.

\section{Definition 3. End-to-end Delay (ED)}

The End-to-end Delay (ED) of a workflow $G_{w}\left(V_{w}, E_{w}\right)$ that is mapped to a computer network $G_{c}$ by $M_{G_{w} \rightarrow G_{c}}$ and scheduled by $S_{G_{w} \rightarrow G_{c}}$, denoted as $E D_{\left\langle G_{w}, G_{c}, M, S\right\rangle}$, is the time duration from the time point when the first module $w_{0}$ in $G_{w}$ starts execution to the time point when the last module $w_{\left|V_{w}\right|-1}$ in $G_{w}$ is finished.

We tabulate the notations used in the cost models in Table 1 for convenience, some of which are used in the algorithm design in Section 4.

\subsection{Workflow Optimization Problem}

We formally define a generic End-to-end Workflow Optimization Problem (EWOP) that considers both mapping and scheduling as follows. 


\section{Definition 4. End-to-end Workflow Optimization Problem (EWOP)}

Given a DAG-structured computing workflow $G_{w}\left(V_{w}, E_{w}\right)$, and a heterogeneous overlay computer network $G_{c}\left(V_{c}, E_{c}\right)$, we wish to find a mapping scheme $M_{G_{w} \rightarrow G_{c}}$ together with an overall on-node scheduling policy $S_{G_{w} \rightarrow G_{c}}$ that achieves the minimum $E D_{\left\langle G_{w}, G_{c}, M, S\right\rangle}$.

The general workflow mapping problem is well known to be NP-complete, and so is the on-node scheduling problem [13]. As a combined optimization problem, EWOP with preemptive scheduling is also NP-complete [45].

We would like to point out that for workflow execution, different stakeholders have different interests, which may not be always aligned well with each other. From the perspective of end users such as research scientists, the main goal is to execute the workflow as fast as possible to improve the research productivity and efficiency in their domains; while from the perspective of resource owners such as cloud service providers, the main goal is to efficiently utilize (limited) computing resources to satisfy the needs of different computing tasks from different users with different requirements. Furthermore, commercial service providers oftentimes have another goal to maximize their financial profit, which may also be in conflict with the other objectives.

In this paper, our goal is to minimize the workflow execution time and help scientists improve the research efficiency in accomplishing their scientific missions. In a typical context for scientific computing, the computing resource is either owned by the institution where the end user is employed or pre-allocated before the actual execution of the workflow. Under these circumstances, the other concerns such as resource cost or utilization may not be as critical as the mission of the research.

\section{Algorithm Design}

We design a Mapping and Scheduling Interaction (MSI) algorithm to solve EWOP, which optimizes workflow end-to-end delay by exploring the interactions between workflow mapping and on-node scheduling. We use a top-down approach to present the MSI algorithm: first describe the overall algorithm structure and then provide design details for each component algorithm. 


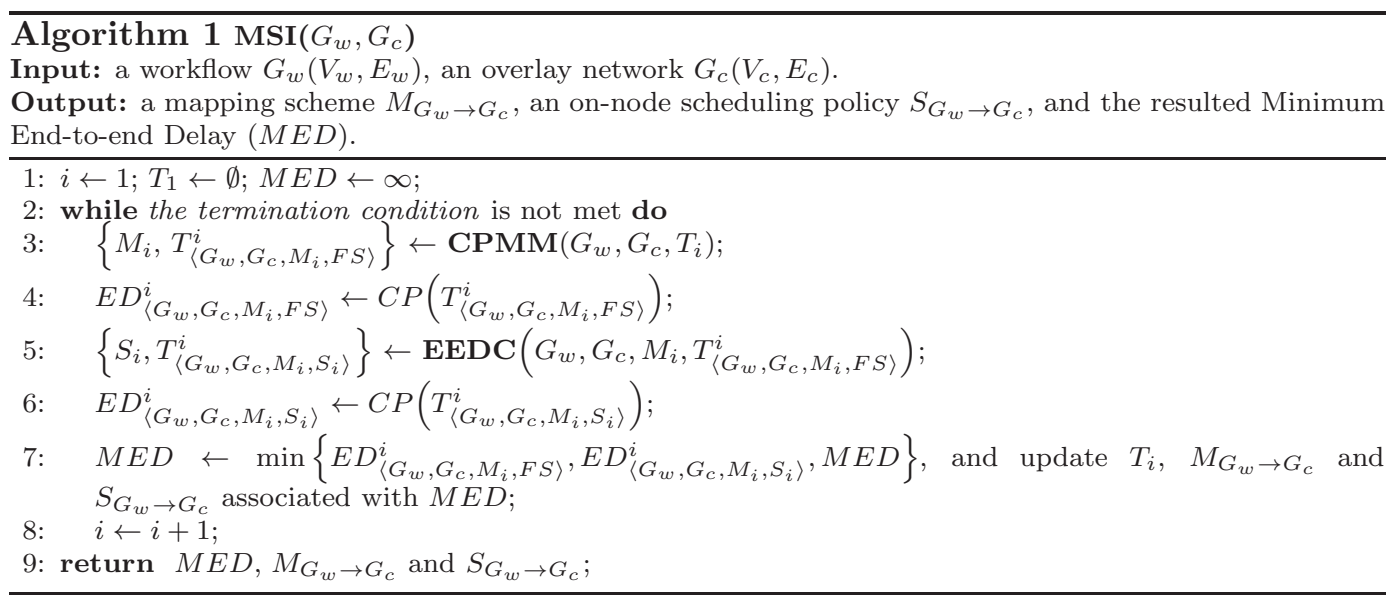

\subsection{Mapping and Scheduling Interaction Algorithm}

As shown in Alg. 1, the MSI algorithm conducts an optimization process in a repetitive manner. In Line 3, MSI first calculates an initial mapping scheme that maps all modules to the network using the Critical Path-based Module Mapping (CPMM) algorithm (Alg. 2 in Section 4.2), which in turn uses the Efficient Linear Pipeline Configuration (ELPC) algorithm [47] (Section 4.2.1) for critical module mapping and the Greedy Branch Module Mapping (GBMM) algorithm (Alg. 3 in Section 4.2.2) for branch module mapping. CPMM yields a mapping scheme $M_{i}$ and calculates the time cost components $T_{\left\langle G_{w}, G_{c}, M_{i}, F S\right\rangle}^{i}$, based on which, MSI computes the critical path $C P\left(T_{\left\langle G_{w}, G_{c}, M_{i}, F S\right\rangle}^{i}\right)$ and the corresponding endto-end delay $E D_{\left\langle G_{w}, G_{c}, M_{i}, F S\right\rangle}^{i}$ in Line 4. In Line 5, MSI determines an on-node job scheduling policy $S_{i}$ using the Exact End-to-end Delay Calculation (EEDC) algorithm (Alg. 4 in Section 4.3), which invokes the Critical Path-based Priority Scheduling (CPPS) algorithm (Alg. 5 in Section 4.4) to perform on-node scheduling on each Concurrent Module Set (CMS), and then calculates the execution time components $T_{\left\langle G_{w}, G_{c}, M_{i}, S_{i}\right\rangle}^{i}$ based on the current schedule $S_{i}$. After calculating the end-to-end delay in Line 6, MSI determines the current best (minimum) End-to-end Delay ( $M E D)$ obtained by the current best mapping scheme $M_{G_{w} \rightarrow G_{c}}$ and on-node scheduling policy $S_{G_{w} \rightarrow G_{c}}$, and calculates the corresponding time cost components $T_{i}$ for the next round of interaction (Line 7 and Line 8). MSI repeats the above process until a certain termination condition is met, for example, when it reaches a pre-specified number of iterations or the performance improvement between two adjacent iterations does not exceed a preset threshold. Particularly, in our implementation, we employ a simple but effective method to control the termination condition of MSI, which involves two constant parameters: $I_{n o \_i m p}$ that denotes the number of contiguous interactions where no improvement is observed over the preceding one and $I_{\max }$ that denotes the maximum number of interactions for a guaranteed termination. The algorithm terminates 


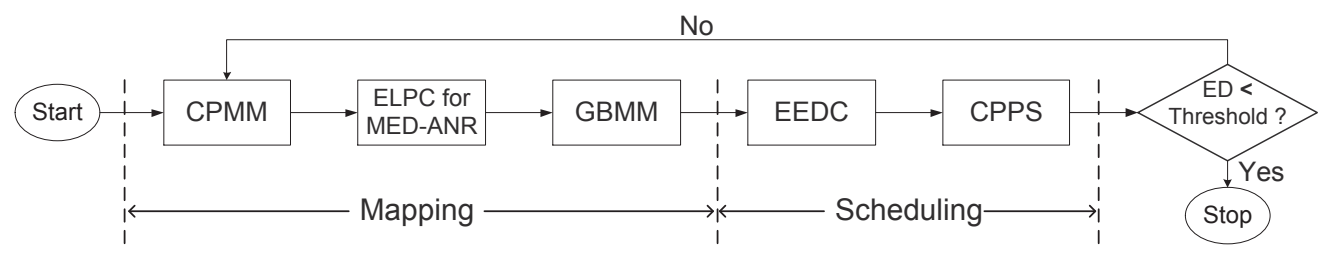

Figure 4: A high-level flowchart of the MSI algorithm.

whichever constant is reached first. These two parameters are customizable and could be adjusted for different applications.

A high-level flowchart of the MSI algorithm is provided in Fig. 4.

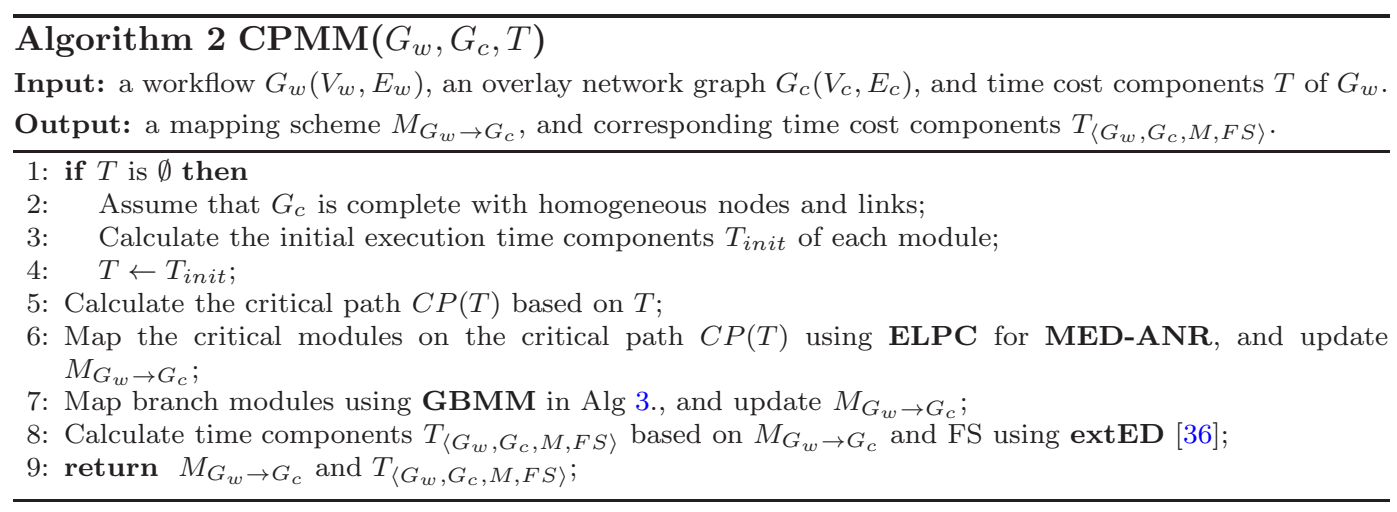

\subsection{Critical Path-based Module Mapping Algorithm}

The Critical Path-based Module Mapping (CPMM) algorithm first maps all the modules on the critical path (i.e. critical modules) to the network using the ELPC algorithm in [49] (Line 6 in Alg. 2), and then uses a greedy approach, Greedy Branch Module Mapping (GBMM) (Line 7 in Alg. 2) to decide the mapping scheme for the workflow modules that are not on the critical path (i.e. branch modules). We describe these two algorithms in Section 4.2.1 and Section 4.2.2, respectively.

\subsubsection{ELPC Algorithm for Critical Module Mapping}

The critical module mapping problem is formally defined as a Minimum End-to-end Delay with Arbitrary Node Reuse (MED-ANR) problem in [49]. Efficient Linear Pipeline Configuration (ELPC) in [49] are a set of algorithms for pipeline mapping in different problem scenarios, among which the optimal one solves MED-ANR problem using a dynamic programming (DP) approach. MSI incorporates ELPC for MED-ANR to determine the mapping scheme for critical modules (Line 6 in Alg. 2). Note that in the initial phase, CPMM assumes that the network is fully-connected with homogeneous computer nodes to calculate the initial time cost components of each module as well as the initial critical path 
and the corresponding end-to-end delay, as shown from Line 1 to Line 5 in Alg. 2. After the first round of interaction, CPMM removes this assumption and uses the updated time cost components for later interactions.

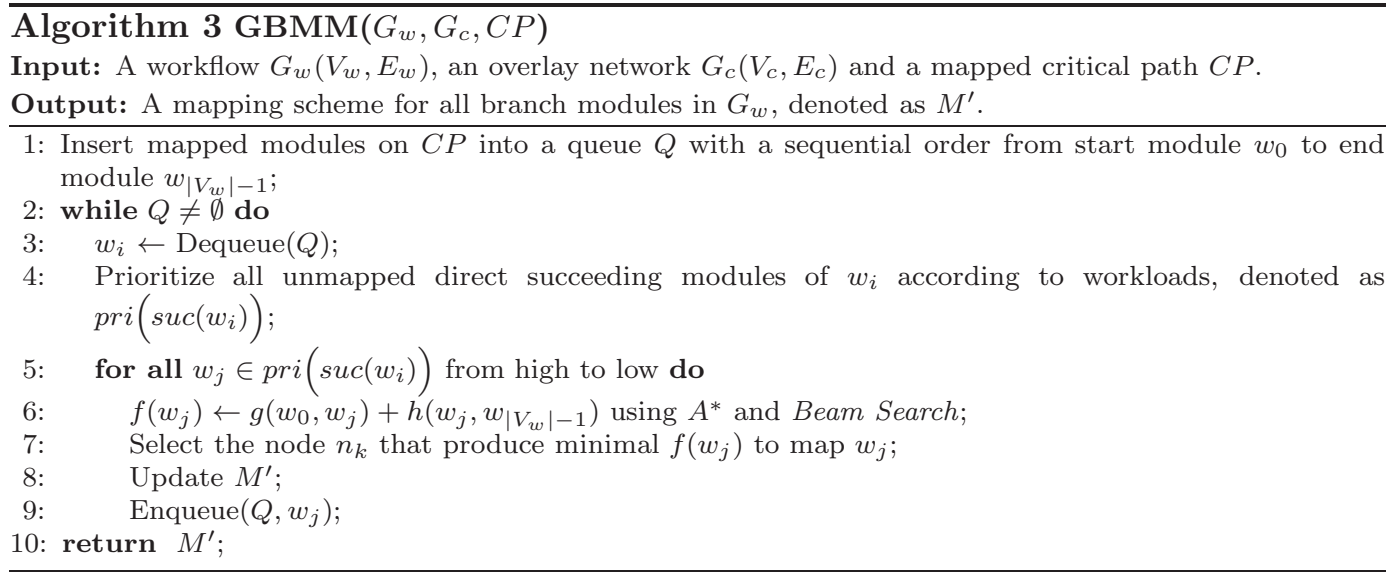

\subsubsection{Greedy Branch Module Mapping Algorithm}

Once the mapping scheme for critical modules is determined, CPMM uses Greedy Branch Module Mapping (GBMM) algorithm to compute the mapping scheme for branch modules (see Line 7 in Alg. 2). The pseudocode of GBMM is shown in Alg. 3, which uses a sortingbased breath first search approach to compute the mapping scheme for branch modules. To improve the naive greedy approach, GBMM employs a cost function $f\left(w_{i}\right)=g\left(w_{0}, w_{i}\right)+$ $h\left(w_{i}, w_{\left|V_{w}\right|-1}\right)$ for module $w_{i} \in G\left(E_{w}, V_{w}\right)$ in $A^{*}$ and Beam Search, which contains two parts: (i) a path cost function $g\left(w_{0}, w_{i}\right)$, calculated from the source node to the current node, which may or may not be optimal; and (ii) a heuristic estimation function $h\left(w_{i}, w_{\left|V_{w}\right|-1}\right)$ of the cost from the current node to the destination node. The greedy procedure of GBMM in the calculation of $h\left(w_{i}, w_{\left|V_{w}\right|-1}\right)$ in Alg. 3 always maps the current module to the computer node that produces the minimum execution time at the current stage without considering whether this would affect the future mapping or the end-to-end delay. To reduce the complexity of the search algorithm, we also explore a predetermined parameter $\lambda$ as the least-cost path in the search tree of the solution space, instead of searching all possible paths. 


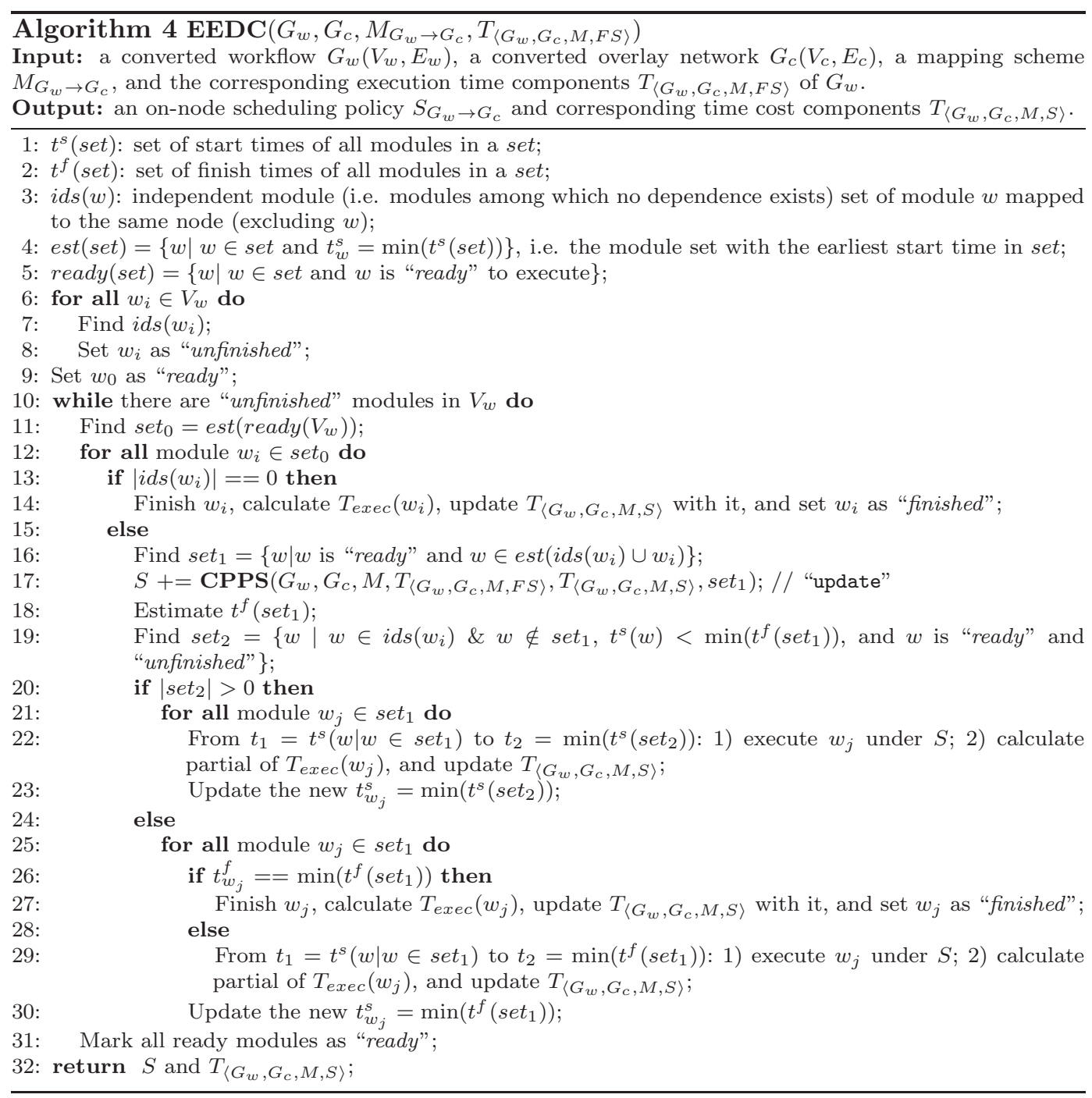

We design an on-node Exact End-to-end Delay Calculation (EEDC) algorithm to perform on-node scheduling and calculate module execution time components in MSI. The EEDC algorithm is a modified version of the extED algorithm in [36] with an integrated on-node scheduling procedure CPPS shown in Alg. 5. It produces a set of processor scheduling policies for all mapping nodes that collectively cut down the execution time of the critical path in the workflow to achieve MED.

The pseudocode of the EEDC algorithm is provided in Alg. 4. Under the given mapping scheme $M_{G_{w} \rightarrow G_{c}}$ (calculated by Alg. 2), it first calculates an independent set (see Line 3 in Alg. 4 for definition) of each module (from Line 6 to Line 8), and then find all modules with earliest start time, as $\operatorname{set}_{0}$ (see Line 11). If there is only one module in set $_{0}$, this module 
is executed immediately to its completion; otherwise, an on-node scheduling strategy over all the concurrent modules in set t $_{0}$ needs to be decided. In Line 16, we find the concurrent module set (CMS) set ${ }_{1}$ with the earliest start time. The CMS of a module $w$, denoted as $\operatorname{CMS}(w)$, is a set that contains all the modules that are mapped to the same node as $w$, and executed concurrently with resource contention. According to Alg. 5, we recalculate the share quantum of the processing power $\operatorname{per}(w)$ that is allocated to each module $w$ in set $_{1}$ in Line 17 . Note that a complete on-node scheduling policy is comprised of all on-node scheduling policies of all the concurrent module sets on their corresponding mapping nodes, which are determined in each while-loop (Line 10). We use "+=" in Line 17 to denote the "update" operation so that at the end, a complete on-node scheduling policy $S_{G_{w} \rightarrow G_{c}}$ for all the modules on their corresponding mapping nodes is returned. Once the on-node scheduling policy is decided, this new on-node scheduling policy is applied to all concurrent modules for their executions and time cost calculations (from Line 18 to Line 30).

\subsection{Critical Path-based Priority Scheduling Algorithm}

We design a Critical Path-based Priority Scheduling (CPPS) algorithm in Alg. 5 to perform the actual on-node scheduling for each CMS. The module execution in EWOP is preemptive and hence leads to complex resource sharing dynamics. We first provide an in-depth analysis of resource sharing.

\subsubsection{Analysis of Resource Sharing Dynamics}

The end-to-end delay of a workflow is determined by its critical path. In general, to minimize the end-to-end delay, we need to allocate more resources to critical modules than those branch modules when they are in the same CMS. In EWOP, the module execution is preemptive, which results in more complex resource sharing dynamics. We present three lemmas on the resource sharing dynamics among the concurrent modules assigned to the same node i.e., within a CMS, which provide us the guidelines in the design of our scheduling algorithm.

Lemma 1. The minimal finish time of a CMS consisting of $k$ concurrent modules executed on a single fully-utilized processor with processing power $P P$ is a constant, $\sum_{i=1}^{k} C R_{i} / P P$.

Proof. Since the total workload of all $k$ modules is fixed, i.e. $\sum_{i=1}^{k} C R_{i}$, and the processor is fully operating, no matter how the modules are scheduled, the total execution time would remain unchanged and is always bounded by the finish time of the last completed module.

To better understand the significance of on-node scheduling during workflow execution, we investigate a typical scheduling scenario shown in Fig. 5 where the best performance 


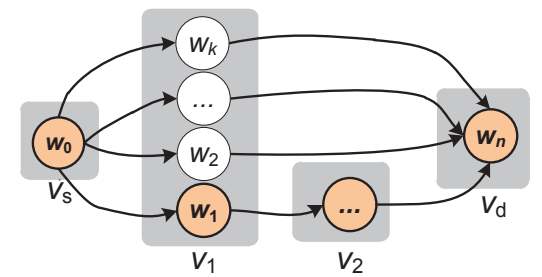

Figure 5: A scheduling scenario that achieves the upper bound performance.

improvement could be achieved over a fair-share scheduling policy under the following conditions:

- There are $k$ modules running concurrently on the same node $v_{1}$ during their entire execution time periods;

- Among $k$ modules, one is a critical module and the other $k-1$ are branch modules, and all of them are of the same workload;

- Each branch module is the only module on its execution path (except for the start and end modules).

Based on the scheduling scenario in Fig. 5, we have Lemma 2:

Lemma 2. The MED performance improvement of any workflow with a fixed critical path achievable by any on-node scheduling over fair-share scheduling is upper bounded by 50\%.

Proof. We first justify that the conditions in Fig. 5 are necessary for achieving the upper bound of the workflow's MED improvement.

Let $x$ be the original execution time of the critical module on node $v_{1}$ using the fairshare scheduling policy. If there were any non-critical modules on $v_{1}$ that finished before $x$, even if we let the critical module $w_{1}$ run exclusively first, it would take longer than $x / k$ to complete. Thus, we would not be able to reduce the execution time of the critical module to the minimum time possible, i.e. $x / k$. On the other hand, if there were any non-critical modules on $v_{1}$ that finished after $x$, from Lemma 1 , we know that the total execution time on this node would be greater than $x$, and hence we would not be able to reduce the length of the critical path to $x$.

The above discussion concludes that all $k$ modules on node $v_{1}$ must share resources during their entire execution time and finish at the same time $x$ to achieve the maximum possible reduction on the length of the critical path. Since we consider fair-share as the comparison base in Lemma 1 , the CRs of all $k$ modules must also be identical. In this scheduling scenario, if we let the critical module $w_{1}$ execute exclusively first, its finish time $t_{w_{1}}^{f}$ would be reduced from $x$ to $x / k$, which is the largest possible improvement with $k$ 


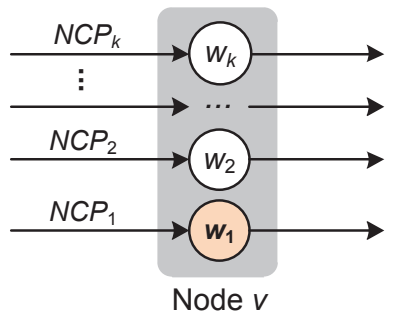

Figure 6: The optimal condition: equally balanced among all NCPs.

concurrent tasks on the same node.

We use $y$ to denote the sum of the execution time for the rest of the modules on the critical path (excluding $\left.w_{1}\right)$. From Lemma 1 , we have $\max \left(t_{w_{i}}^{f}\right)=x,(i=2,3, \cdots, k)$, which means that the latest finish time of the last completed branch module would be still $x$. Since the new length of the critical path (i.e. MED) becomes $(x / k)+y$, the upper bound MED improvement is achieved if this new length is equal to the latest finish time of any branch module, i.e. $(x / k)+y=x$. It follows that $y=((k-1) / k) \times x$. Therefore, the MED improvement over fair share is $\Delta=((x+y)-x) /(x+y)=(k-1) /(2 k-1)$, which is $50 \%$ as $k \rightarrow \infty$.

We define several key terms, which are used in Lemma 3.

Definition 5. The execution time of a module $w$ is the time duration starting from the time point when $w$ becomes "ready" to the time point when $w$ finishes its execution completely.

In our model, $w$ is executed immediately once it becomes "ready" unless the scheduling policy suspends it and allocates computing resources to other concurrent modules. According to Def. 5, w's execution time includes its waiting time.

Definition 6. The Global Critical Path (GCP) of a DAG-structured workflow $G_{w}\left(V_{w}, E_{w}\right)$ is the longest path of execution time from the start module $w_{0}$ to the end module $w_{\left|V_{w}\right|-1}$.

Definition 7. The Nodal Critical Path (NCP) of module w in a DAG-structured workflow is the longest path of execution time traversing module $w$.

Based on Lemma 1 and Lemma 2, we have the following lemma to illustrate the condition, under which an optimal scheduling solution is achievable.

Lemma 3. The MED of a DAG-structured workflow is achieved if and only if when the Nodal Critical Paths of all workflow modules that share resources with the critical modules are scheduled to have the same length as the Global Critical Path. 
Proof. Without loss of generality, we consider a workflow where a concurrent module set $\left\{w_{1}, w_{2}, \cdots, w_{k}\right\}$ is mapped to the same node $v$, as shown in Fig. 6 . Suppose that the nodal critical path $N C P\left(w_{1}\right)$ is the global critical path of the workflow. Branch modules $w_{2}, \cdots, w_{k}$ are mapped to the same node $v$ with resource competition. There exists $k$ NCPs that go through $v$, denoted as $N C P_{1}, N C P_{2}, \cdots, N C P_{k}$, with their lengths denoted as $l_{1}, l_{2}, \cdots, l_{k}$, respectively (assume that $l_{1} \geq l_{2} \geq \cdots \geq l_{k}$ ). If they are not of equal length, since $N C P_{1}$ is the longest, one can always allocate more resource of $v$ to the critical module $w_{1}$ such that $l_{1}$ would become shorter and the other NCPs would become longer.

If we move resources to the critical module from those branch modules in some reasonable manner, theoretically, we should be able to obtain a new mapping and scheduling strategy where all NCPs are equal with a new length $l_{e q}$, where $l_{1} \leq l_{e q} \leq l_{k}$. The same is also true for other critical modules that share resources with branch modules.

\subsubsection{Design of $C P P S$}

The key idea of CPPS is to "squeeze" an appropriate amount of computing resources out of branch modules and re-allocate them to critical modules. Towards this end, a critical task is to decide the percent $\operatorname{per}\left(w_{i}\right)$ of CPU allocation for each module $w_{i}$ in the concurrent module set $\operatorname{CMS}\left(w_{i}\right)$.

CPPS schedules all concurrent module sets in a left-to-right manner based on the execution time components under fair-share as well as the partial execution time components under the new on-node scheduling policy. In Alg. 5, from Line 8 to Line 10, it first checks all the modules in the CMS: if there is no critical module, a fair-share schedule is simply returned (from Line 11 to Line 13); otherwise, CPPS attempts to let the critical modules execute with more processing power reallocated from those branch modules in CMS. If such a new on-node scheduling does not change the original critical path of the workflow, CPPS returns this schedule; otherwise, it estimates the maximal amount of processing power that can be moved from the branch modules to the critical modules, as shown from Line 14 to Line 34 .

To estimate the amount of processing power for reallocation, CPPS first estimates the execution times $T_{E X}$ of critical modules if they are executed exclusively (from Line 14 to Line 15). Since multiple NCPs may have the same length, if there are multiple critical modules, they share the processing power in a fair manner. In Line 16, the latest finish time lft $(v)$ of each module mapped onto node $v$ is calculated according to Lemma 1. For each branch module $w_{i}$ (Line 18), we estimate the time $T_{\text {exec }}\left(t b f\left(w_{i}\right)\right)$ it needs to finish (Line 19), and the amount of time that can be possibly delayed, denoted as $\delta t_{1}$ and $\delta t_{2}$ (Line 20 
and Line 21). We extend the execution time of the branch module by $\delta t_{1}$ or $\delta t_{2}$ (Line 24), whichever has a lower possibility to change the original critical path. If this extension does not change the original critical path based on the estimation indicated by $\beta$ in Line 22 , the share quantum per $\left(w_{i}\right)$ of processing power allocated to each module $w_{i}$ is calculated based on $\Delta t$ (from Line 23 to Line 26). Otherwise, we mark it using a boolean variable flag (Line $28)$.

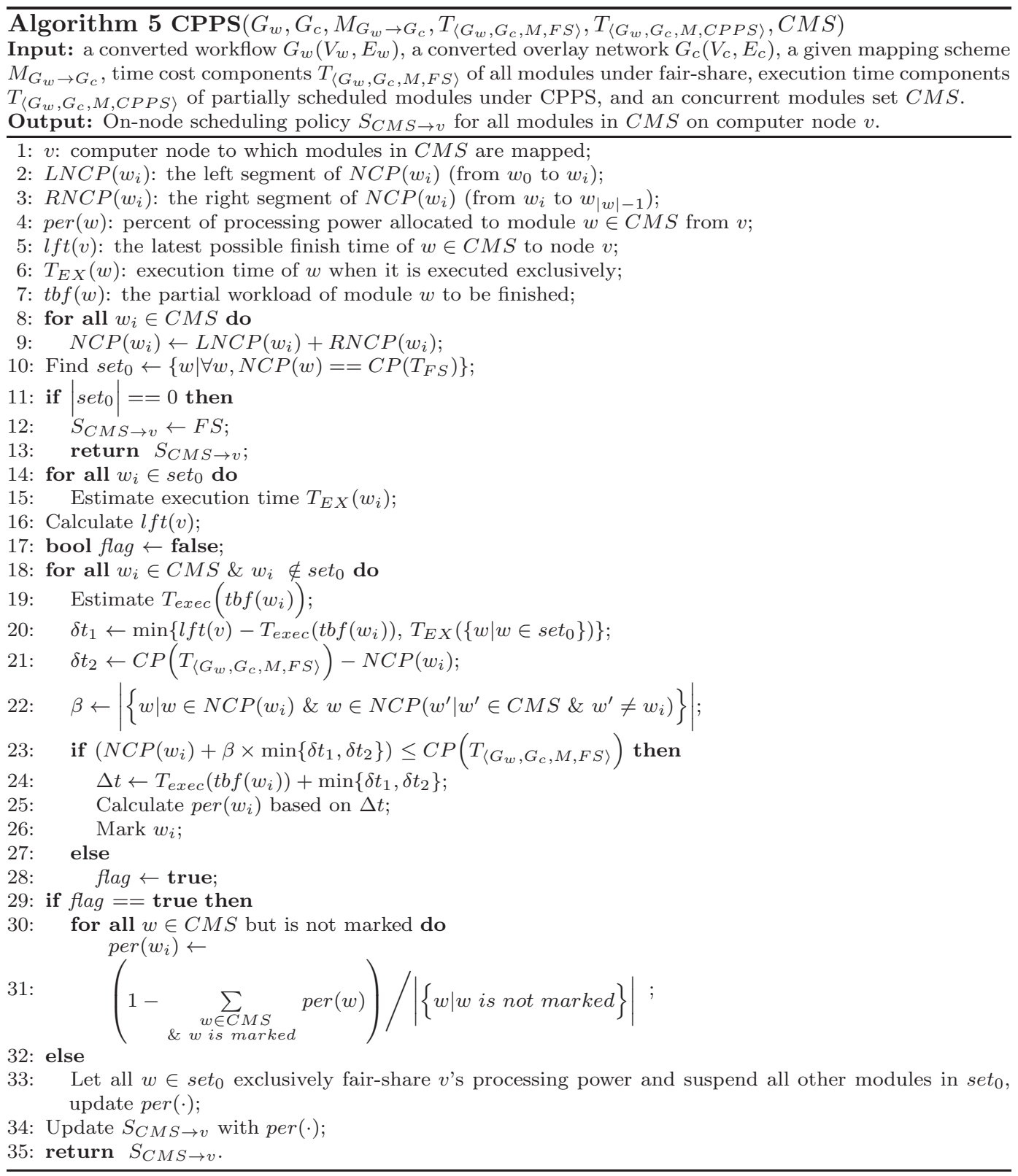

To further explain how $\delta t_{1}$ and $\delta t_{2}$ are calculated, we consider a CMS with three modules $w_{1}, w_{2}$ and $w_{3}$ that are mapped to a computer node $v$. These three modules start executing 


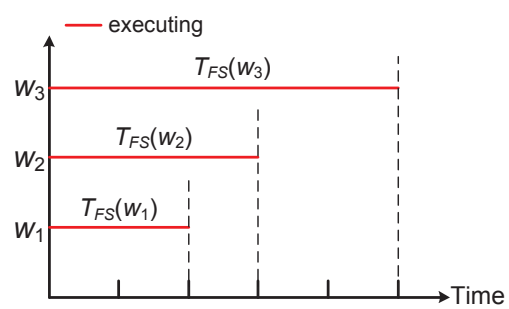

(a)

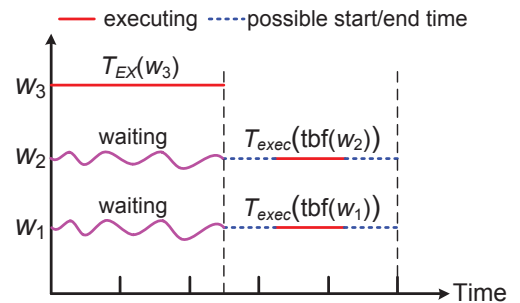

(b)

Figure 7: Illustration of parameters $\delta t_{1}$ and $\delta t_{2}$ in Alg. 5 .

at the same time, and module $w_{3}$ is the critical module, which is the last one to be finished. Fig. 7(a) shows the execution dynamics of this CMS under fair-share scheduling. If we let $w_{3}$ execute exclusively ahead of $w_{1}$ and $w_{2}$, the execution dynamics are shown in Fig. 7(b), where dotted lines represent possible start and finish time points of $w_{1}$ and $w_{2}$, which depend on the workloads of $w_{1}$ and $w_{2}$, the processing power of $v$, and the specific on-node scheduling policy on the CMS. No matter how their executions are scheduled, either $w_{1}$ or $w_{2}$, or both of them would finish at the latest possible finish time $T_{\text {exec }}(t b f(v))$ according to Lemma 1. Therefore, for each branch module $w_{i} \in\left\{w_{1}, w_{2}\right\}$, in general, we have the following inequality,

$$
T_{\text {exec }}\left(t b f\left(w_{i}\right)\right)+\delta t_{1} \leq l f t(v)
$$

where $\delta t_{1}$ is the amount of execution time that can be delayed for the branch module. If $w_{3}$ is scheduled for exclusive execution ahead of $w_{1}$ and $w_{2}$, we have

$$
\begin{gathered}
\delta t_{1}=T_{E X}\left(w_{3}\right), \\
T_{\text {exec }}\left(t b f\left(w_{i}\right)\right)+T_{E X}\left(w_{3}\right) \leq l f t(v)(i=1,2) .
\end{gathered}
$$

To avoid critical path shifting, which may lead to a longer end-to-end delay, the execution time of the branch module cannot be overly extended. Hence, we calculate $\delta t_{1}$ as follows (Line 20):

$$
\delta t_{1}=\min \left\{l f t(v)-T_{\text {exec }}\left(t b f\left(w_{i}\right)\right), T_{E X}\left(w_{3}\right)\right\} .
$$

Also, any delay on the finish time of branch module $w_{i}$ would also increase the length of $\operatorname{NCP}\left(w_{i}\right)$. For $w \in\left\{w_{1}, w_{2}\right\}$, we further avoid critical path shifting by imposing the following conditions to calculate $\delta t_{2}$ :

$$
N C P(w)+\delta t_{2} \leq G C P(w)=C P\left(T_{\left\langle G_{w}, G_{c}, M, F S\right\rangle}\right)
$$




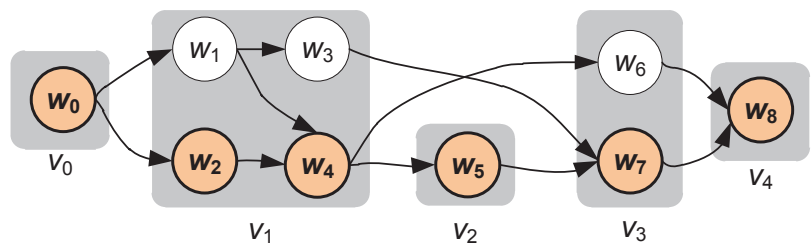

Figure 8: A simple numerical example used for illustrating the CPPS scheduling algorithm.

$$
\delta t_{2} \leq C P\left(T_{\left\langle G_{w}, G_{c}, M, F S\right\rangle}\right)-N C P(w)
$$

Since different NCPs and the GCP of the workflow may share some modules, the execution time extension of branch modules may affect the workflow's GCP. Thus, in Line 23, we check if the extension changes the original critical path $C P\left(T_{\left\langle G_{w}, G_{c}, M, F S\right\rangle}\right)$. $\beta$ in Line 22 is the number of the common modules that the workflow's GCP and all independent modules' NCPs share. If this extension does not change the original critical path, we extend the amount of execution time for branch modules by $\min \left\{\delta t_{1}, \delta t_{2}\right\}$, and then use flag to indicate whether there are some branch modules that may change the GCP (Line 28). If flag is changed to be true, we let all critical modules and those marked modules (Line 26) fairly share the node's processing power with the amount reallocated from the branch modules (from Line 29 to Line 31); otherwise, since GCP is not changed by the new on-node scheduling policy, we let the critical module exclusively execute before the branch modules (from Line 32 to Line 33).

The on-node scheduling policy $S_{C M S \rightarrow v}$ is returned as a vector $\operatorname{per}(\cdot)$ in Alg. 5, where we consider two cases:

- When $\operatorname{per}\left(w \mid w \in \operatorname{set}_{0}\right)=1$ and $\operatorname{per}\left(w \mid w \notin \operatorname{set}_{0}\right)=0$ : we exclusively execute the critical module until some module finishes (where a new on-node scheduling policy $S^{\prime}$ is needed); while for the other modules, we suspend them to wait for the critical module to finish. During this period of time, the critical module may be entirely or partially completed. We then execute all the modules (their unfinished part $t b f\left(w_{i}\right)$ ) that are mapped to node $v$ based on the new on-node scheduling policy $S^{\prime}$ until the number of concurrent modules assigned to $v$ changes again.

- When all $\operatorname{per}(\cdot)$ are between 0 and 1: we execute each module with its own share quantum $\operatorname{per}(\cdot)$ of resource allocation until the number of concurrent modules changes.

We provide a simple numerical example to illustrate the CPPS scheduling process. As shown in Fig. 8 , a computing workflow $G_{w}$ consisting of 9 modules $w_{0}, w_{1}, \ldots, w_{8}$ is mapped 


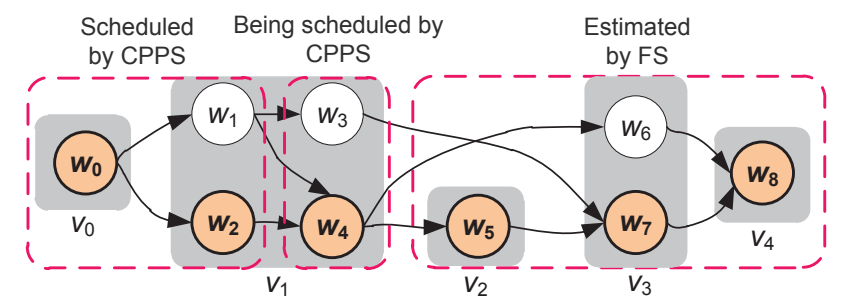

Figure 9: The snapshot when $w_{3}$ and $w_{4}$ (middle circled part) are being scheduled by CPPS. The left circled part has been scheduled by CPPS while the right circled part is estimated based on FS.

Table 2: The module execution time in the example workflow under FS and CPPS.

\begin{tabular}{|l|c|c|c|c|c|c|c|c|c|}
\hline Module & $w_{0}$ & $w_{1}$ & $w_{2}$ & $w_{3}$ & $w_{4}$ & $w_{5}$ & $w_{6}$ & $w_{7}$ & $w_{8}$ \\
\hline Workload (units) & 0 & 10 & 40 & 20 & 10 & 80 & 60 & 40 & 0 \\
\hline Mapped to & $v_{0}$ & $v_{1}$ & $v_{1}$ & $v_{1}$ & $v_{1}$ & $v_{2}$ & $v_{3}$ & $v_{3}$ & $v_{4}$ \\
\hline Start time (FS) & $0 \mathrm{~s}$ & $0 \mathrm{~s}$ & $0 \mathrm{~s}$ & $2 \mathrm{~s}$ & $7 \mathrm{~s}$ & $8 \mathrm{~s}$ & $8 \mathrm{~s}$ & $16 \mathrm{~s}$ & $20 \mathrm{~s}$ \\
\hline End time (FS) & $0 \mathrm{~s}$ & $2 \mathrm{~s}$ & $7 \mathrm{~s}$ & $6 \mathrm{~s}$ & $8 \mathrm{~s}$ & $16 \mathrm{~s}$ & $14 \mathrm{~s}$ & $20 \mathrm{~s}$ & $20 \mathrm{~s}$ \\
\hline Start time (CPPS) & $(0 \mathrm{~s})$ & $(0 \mathrm{~s})$ & $(0 \mathrm{~s})$ & $(5 \mathrm{~s})$ & $(5 \mathrm{~s})$ & $8 \mathrm{~s}$ & $8 \mathrm{~s}$ & $16 \mathrm{~s}$ & $20 \mathrm{~s}$ \\
\hline End time (CPPS) & $(0 \mathrm{~s})$ & $(5 \mathrm{~s})$ & $(4 \mathrm{~s})$ & $(8 \mathrm{~s})$ & $(6 \mathrm{~s})$ & $16 \mathrm{~s}$ & $14 \mathrm{~s}$ & $20 \mathrm{~s}$ & $20 \mathrm{~s}$ \\
\hline Start time (CPPS) & $(0 \mathrm{~s})$ & $(0 \mathrm{~s})$ & $(0 \mathrm{~s})$ & $(5 \mathrm{~s})$ & $(5 \mathrm{~s})$ & $(6 \mathrm{~s})$ & $(6 \mathrm{~s})$ & $(14 \mathrm{~s})$ & $(18 \mathrm{~s})$ \\
\hline End time (CPPS) & $(0 \mathrm{~s})$ & $(5 \mathrm{~s})$ & $(4 \mathrm{~s})$ & $(8 \mathrm{~s})$ & $(6 \mathrm{~s})$ & $(14 \mathrm{~s})$ & $(12 \mathrm{~s})$ & $(18 \mathrm{~s})$ & $(18 \mathrm{~s})$ \\
\hline
\end{tabular}

to a computer network $G_{c}$ consisting of 5 nodes $v_{0}, v_{1}, \ldots, v_{4}$. The workloads of $G_{w}$ and the mapping scheme for $G_{w}$ are tabulated in Table 2. The processing powers of the computer nodes are $P P_{1}=10 \mathrm{units} / \mathrm{s}, P P_{2}=10 \mathrm{units} / \mathrm{s}$, and $P P_{3}=10 \mathrm{units} / \mathrm{s}$, respectively. Under a fair-share (FS) scheduling policy, the start time and end time of each module are provided in Table 2, where $G_{w}$ takes 20 seconds to complete along the critical path consisting of modules: $w_{0} \rightarrow w_{2} \rightarrow w_{4} \rightarrow w_{5} \rightarrow w_{7} \rightarrow w_{8}$.

For CPPS, Fig. 9 illustrates the scheduling status when modules $w_{3}$ and $w_{4}$ are being scheduled by CPPS in Alg. 5. At this point of time, the left part has been scheduled by CPPS while the right shadowed part is estimated based on FS (we tabulate the execution time in Table 2 and use parentheses to differentiate those that have been produced by CPPS). Note that the module's execution start time is always calculated from the point when the module is "ready" to execute, not from the point when it actually starts running. Right after $w_{1}$ finishes execution, both $w_{3}$ and $w_{4}$ are "ready" to execute with potential resource competition. To decide the sharing policy between them, we need to compute the critical path that traverses each of them (NCP) by concatenating the left and right segments of its critical path. For $w_{3}$, the length of its left CP segment $\left(w_{0} \rightarrow w_{1} \rightarrow w_{3}\right)$, denoted as $\operatorname{LNCP}\left(w_{3}\right)$, is 5 under CPPS, while the length of its right CP segment $\left(w_{3} \rightarrow w_{7} \rightarrow w_{8}\right)$, denoted as $R N C P\left(w_{3}\right)$, is 8 including $w_{3}$ 's own execution time, which is estimated based on FS. Similarly, for $w_{4}$, the lengths of its $L N C P\left(w_{4}\right)$ and $\operatorname{RNCP}\left(w_{4}\right)$ are 5 under CPPS and 
13 under FS, respectively. Since $N C P\left(w_{4}\right)=\operatorname{LNCP}\left(w_{4}\right)+R N C P\left(w_{4}\right)=18$ is longer than $N C P\left(w_{3}\right)=\operatorname{LNCP}\left(w_{3}\right)+R N C P\left(w_{3}\right)=13, w_{4}$ is to be executed with more computing resource. We estimate that the length of $\operatorname{NCP}\left(w_{3}\right)$ would not exceed the length of the original global critical path based on FS, which is 20 , even if we let $w_{4}$ run to its completion with exclusive use of processing power. So, in this case, we assign the entire $P P_{1}$ to $w_{4}$ until it finishes; otherwise, $w_{4}$ would execute exclusively until a certain point and then share with $w_{3}$ in a fair-share manner such that the length of $N C P\left(w_{3}\right)$ does not exceed the length of the original global critical path based on FS. This scheduling process is repeated on every computer node with multiple modules mapped until all the modules are properly scheduled.

\subsection{Time Complexity of MSI}

According to Line 2 and Line 5 in Alg. 3, the time complexity of GBMM is $O\left(\lambda \cdot m \cdot\left|E_{w}\right|\right)$, where $\lambda$ is the predetermined number of least-cost paths to reduce the complexity, $m$ is the number of modules in the workflow, and $\left|E_{w}\right|$ is the number edges in the workflow. According to Lines 6, 7, and 8 in Alg. 2, the time complexity of CPMM is $O\left(\left(\lambda \cdot m \cdot\left|E_{w}\right|\right)+\left(m \cdot\left|E_{w}\right|\right)+\right.$ $\left.\left(m^{3}\right)\right)=O\left(\lambda \cdot m \cdot\left|E_{w}\right|+m^{3}\right)=O\left(\lambda \cdot m^{3}\right)$ since the time complexity of ELPC and extED are $O\left(m \cdot\left|E_{w}\right|\right)$ and $O\left(m^{3}\right)$, respectively $[49,36]$. According to Line 8, Line 14, and Line 18 in Alg. 5, the time complexity of CPPS is $O(\alpha \cdot m)$ since running time complexity of calculating a critical path in a DAG is linear, where $\alpha$ is the number of modules in the CMS. According to Line 10, Line 12, Line 17, Line 21, and Line 25 in Alg. 4, the time complexity of EEDC is $O\left((\alpha \cdot m+m) \cdot m^{2}\right)=O\left(\alpha \cdot m^{3}\right)$.

As such, according to Line 2, Line 3, and Line 5 in Alg. 1, the overall time complexity of MSI is $O\left(k \cdot\left(\lambda \cdot m^{3}+\alpha \cdot m^{3}+m\right)\right)=O\left(k \cdot(\lambda+\alpha) \cdot m^{3}\right)$, where $k$ is the number of iterations, $\lambda$ is a predefined number of least-cost paths, $\alpha$ is the number of modules in the CMS that depends on the workflow structure and specific mapping and scheduling schemes, and $m$ is the size of the workflow (i.e. the number of modules).

\section{Performance Evaluation}

We evaluate the performance of the proposed MSI algorithm in both simulated and experimental environments in comparison with various existing methods.

\subsection{Simulations}

We implement the MSI algorithm in $\mathrm{C}++$ and run it on a Windows 7 desktop PC equipped with Inter(R) Core(TM)2 Duo CPU E7500 of 2.93GHz and 4.00GB memory. 


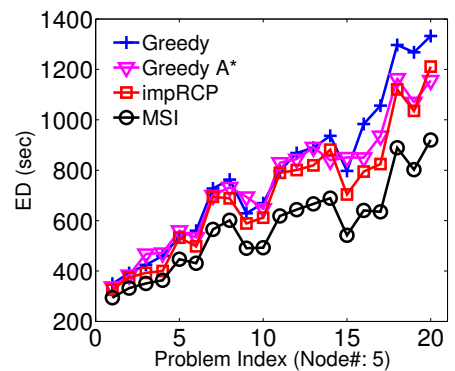

(a)

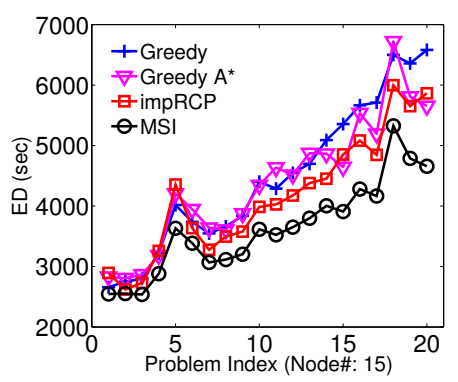

(c)

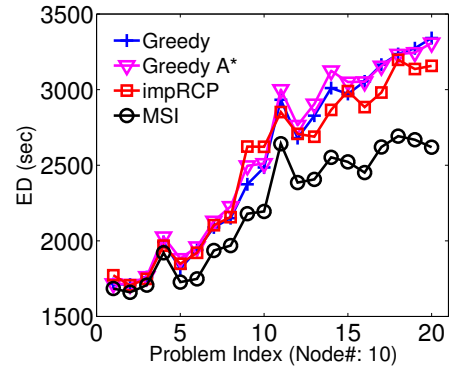

(b)

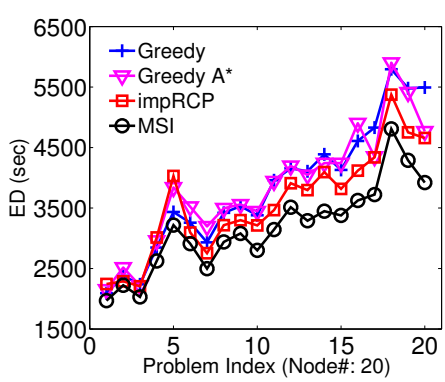

(d)

Figure 10: End-to-end delay (seconds) of MSI and Greedy, Greedy A*, and impRCP.

We adopt a similar program in [36] to generate test datasets in the simulation by varying the parameters of workflows and networks. These parameters are represented by a threetuple $\left\langle m,\left|E_{w}\right|, n\right\rangle: m$ modules and $\left|E_{w}\right|$ edges in workflow, and $n$ nodes in computer network.

\subsubsection{Simulation Results}

To evaluate the performance of MSI, we randomly generate 4 groups of test cases with 4 different numbers of nodes, i.e. $n=5,10,15,20$. In each group, we generate test cases in 20 different problem sizes, indexed from 1 to 20 , by varying the number of modules from 30 to 125 , at an interval of 5 with a random number of edges.

For each problem size $\left\langle m,\left|E_{w}\right|, n\right\rangle$, we generate 20 random problem instances for workflow optimization with different module complexities, data sizes, node processing powers, and link bandwidths, which follow a uniform distribution in an appropriate range of values. We collect the performance measurements for Simple Greedy, Greedy A* [50], impRCP [36], and MSI, and calculate the average of 20 instances in each case. The end-to-end delay comparison between these algorithms is plotted in Fig. 10. The MED performance improvement or speedup of MSI over impRCP, defined as $\frac{E D_{i m p R C P}-E D_{M S I}}{E D_{i m p R C P}} \times 100 \%$, is tabulated in Table 3 and further plotted in Fig. 11 with both the mean and standard deviation for a visual examination. 


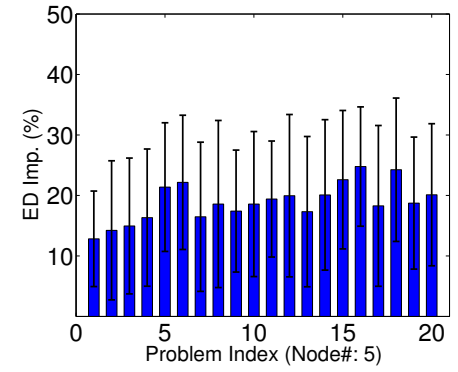

(a)

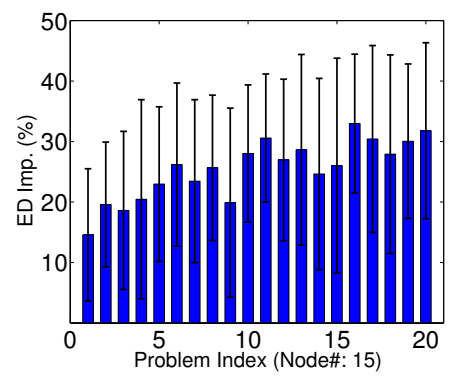

(c)

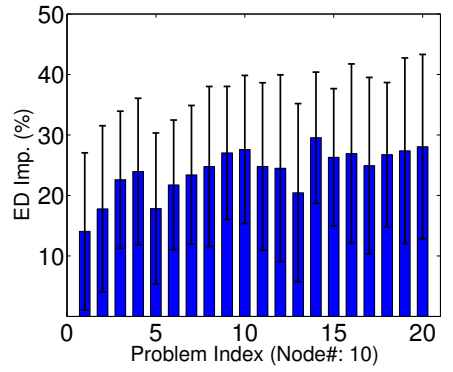

(b)

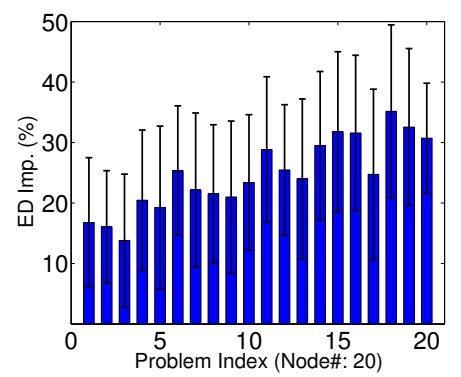

(d)

Figure 11: End-to-end delay improvement (\%) of MSI over impRCP.

Table 3: MED improvement percentage of MSI over impRCP.

\begin{tabular}{|c|c|c|c|c|c|}
\hline Problem & Module \# & \multicolumn{4}{|c|}{ Average MED Improvement (\%) } \\
\cline { 3 - 6 } Index & $m$ & $n=5$ & $n=10$ & $n=15$ & $n=20$ \\
\hline 1 & 30 & 12.83 & 14.06 & 14.56 & 16.79 \\
\hline 2 & 35 & 14.23 & 17.79 & 19.61 & 16.06 \\
\hline 3 & 40 & 14.96 & 22.60 & 18.62 & 13.82 \\
\hline 4 & 45 & 16.34 & 23.96 & 20.46 & 20.47 \\
\hline 5 & 50 & 21.38 & 17.83 & 22.97 & 19.26 \\
\hline 6 & 55 & 22.17 & 21.73 & 26.19 & 25.39 \\
\hline 7 & 60 & 16.48 & 23.41 & 23.45 & 22.17 \\
\hline 8 & 65 & 18.58 & 24.79 & 25.66 & 21.53 \\
\hline 9 & 70 & 17.42 & 27.04 & 19.89 & 21.01 \\
\hline 10 & 75 & 18.58 & 27.60 & 28.03 & 23.41 \\
\hline 11 & 80 & 19.41 & 24.79 & 30.58 & 28.85 \\
\hline 12 & 85 & 19.96 & 24.50 & 26.97 & 25.47 \\
\hline 13 & 90 & 17.32 & 20.44 & 28.63 & 24.00 \\
\hline 14 & 95 & 20.09 & 29.55 & 24.63 & 29.49 \\
\hline 15 & 100 & 22.61 & 26.30 & 26.03 & 31.79 \\
\hline 16 & 105 & 24.78 & 26.92 & 32.98 & 31.58 \\
\hline 17 & 110 & 18.28 & 24.94 & 30.43 & 24.74 \\
\hline 18 & 115 & 24.25 & 26.75 & 27.91 & 35.16 \\
\hline 19 & 120 & 18.74 & 27.38 & 30.05 & 32.55 \\
\hline 20 & 125 & 20.11 & 28.06 & 31.79 & 30.72 \\
\hline
\end{tabular}

In our simulations, we observe that the performance improvement largely depends on the mapping scheme since it determines the resource sharing level during workflow execution. In small problem sizes, or when the number of modules is comparable to the number of nodes, the modules are likely to be mapped to the nodes in a uniform manner, resulting in a 
low level of resource sharing, unless there exist some nodes whose processing power is significantly higher than the others in the network. Hence, in these cases, the MED improvement of MSI over impRCP is not very obvious. However, as the problem size increases, more modules might be mapped to the same node with more resource sharing, leading to a higher performance improvement. This overall trend of performance improvement is reflected in Fig. 10 and Fig. 11.

The amount of resource sharing in the workflow execution depends on not only the number of modules, but also the structure of the workflow and the complexities of modules. For a workflow with a small number of modules in each layer, only a limited amount of resource sharing may occur. If the complexity of a critical module $w$ is significantly larger than that of others that compete for resource, running $w$ exclusively would only yield a marginal performance improvement. This overall trend of performance improvement is also reflected in Fig. 10 and Fig. 11.

The above comparison between MSI and $i m p R C P$ shows that the performance improvement varies significantly from case to case because it highly depends on the level of resource sharing in the test case, as indicated by the standard deviations in Fig. 11. Generally, as long as such resource sharing exists with limited resources, MSI is able to achieve a consistently better end-to-end performance than the other methods.

\subsubsection{MSI Optimization Evolution Process}

MSI alleviates the negative effect of the critical path shifting on workflow mapping caused by on-node scheduling and eventually improves the overall end-to-end performance. Starting from an initial mapping scheme, MSI keeps searching for a relatively "better" critical path by exploring the interactions between mapping and scheduling to optimize the workflow performance.

To examine the microscopic behavior of MSI involving both mapping and scheduling, we look into one typical test case of problem index 14 with parameters $m=95,\left|E_{w}\right|=420$, $n=10$. We plot the performance measurements at all the iterations in Fig. 12, where the iterations with critical path shifting are marked. We observe that the end-to-end delay is continuously improved when such a shifting occurs until the algorithm converges to a stable point.

\subsection{Proof-of-concept Experiments}

To evaluate the efficacy of the MSI algorithm, we apply it to a real-life scientific workflow, Weather Research and Forecasting (WRF) [51], provided by Brookhaven National Laboratory (BNL), as shown in Fig. 13. We compose such a workflow using WPS-WRF modules, 


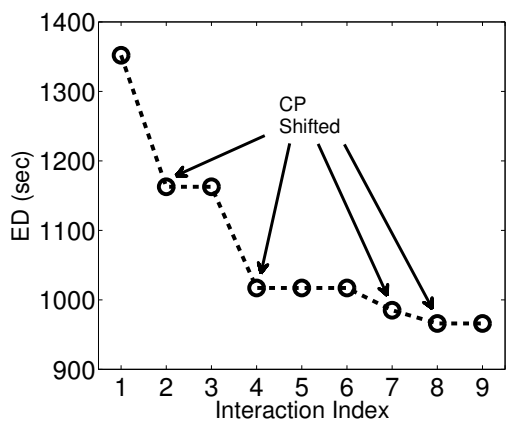

Figure 12: The interaction convergence curve in Experiment 14 with parameters $m=95,\left|E_{w}\right|=420$, $n=10$ in Fig. 10(b).

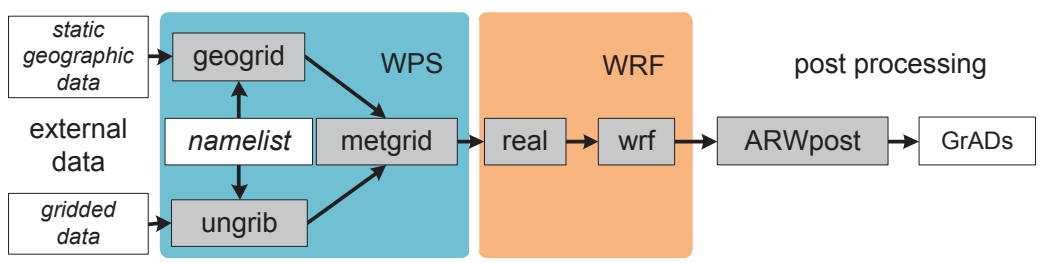

Figure 13: Climate modeling workflow structure.

and map and execute it in a local PC cluster, which contains 16 compute nodes equipped with 12 Intel(R) Xeon(R) CPUs of $1200 \mathrm{MHz}$ and 64G RAM.

\subsubsection{Weather Research and Forecasting (WRF)}

The Weather Research and Forecasting (WRF) model [51] from BNL [52] has been widely used for regional to continental scale weather forecast. The workflow for typical applications of WRF model takes multiple steps, including data preparation and preprocessing, actual model simulation, and postprocessing. Each step could be computationally intensive and/or involve a large amount of data transfer and processing. Moreover, because of the chaotic nature of the atmospheric system and unavoidable errors in the input data and the imperfection of the model, ensemble approaches have to be adopted with a sufficiently large number of simulations. A workflow-based management and execution is hence extremely useful to automate the procedure and efficiently allocate the resources to carry out the required computational tasks.

\subsubsection{Composite Climate Modeling Workflow Structure}

As shown in Fig. 14, we duplicate the entire WPS-WRF workflow to generate five parallel pipelines to process five different dataset instances of the workflow input. The sizes of 5 input datasets are: 423.38 MB (Pipeline 1), 106.15 MB (Pipeline 2), 106.15 MB (Pipeline 3), 106.15 MB (Pipeline 4), and 106.15 MB (Pipeline 5), respectively. By doing so, we 
are able to construct a relatively complex workflow that contains 32 modules. We employ the performance modeling and profiling approach in [53] to determine the workload of each module prior to the actual execution. We map the composite workflow to 10 compute nodes $\left(n_{1}\right.$ to $\left.n_{10}\right)$ in the PC cluster to process five batches of climate modeling input datasets. Since $w_{0}$ and $w_{31}$ are virtual start and end modules, they are not mapped to any physical compute node. Note that each pipeline in Fig. 14 corresponds to the climate modeling workflow shown in Fig. 13, i.e. geogrid $\leftrightarrow w_{1}$, ungrib $\leftrightarrow w_{2}$, metgrid $\leftrightarrow w_{3}$, real $\leftrightarrow w_{4}$, wrf $\leftrightarrow w_{5}$, ARWpost $\leftrightarrow w_{6}$, and so on.

\subsubsection{A PC Cluster-based Workflow Engine}

To automatically execute the entire composite workflow shown in Fig. 14, we develop a lightweight PC Cluster-based Workflow Engine, referred to as ClusterWE, to manage the workflow execution under the inter-module precedence constraints.

ClusterWE is a daemon program that runs on every compute node with at least one workflow module mapped. As shown in Fig. 15, ClusterWE has a controller that includes a sender for sending a finish ("FIN") signal to the succeeding node(s) and a receiver for receiving a finish signal from the preceding node(s), and an executer for scheduling and executing workflow module(s). The controller runs on a different core from the executer to avoid computing resource competition with workflow modules (core 1 and core 2, as shown in Fig. 15). The workflow structure, workflow mapping scheme, and executable list are stored in three different configuration files. Fig. 16 shows the format and contents of the workflow structure file and mapping scheme file. ClusterWE manages the workflow execution as follows:

1. A ClusterWE instance runs on each compute node with module(s) mapped, and parses the workflow structure, mapping scheme and executable information from the configuration files.

2. The receiver of the controller (as a daemon thread) listens on a pre-defined port number for a one-byte finish ("FIN") message that indicates the completion of a preceding module. The delay in transmitting this "FIN" message is considered negligible compared to the inter-module data transfer delay in the workflow.

3. Once all finish messages for one mapped module are received from its preceding modules, this module becomes "ready" and starts running within the executer on another core as a newly created thread. When there exists computing resource competition among multiple modules executed simultaneously (either multiple mapped modules are "ready" or some modules are already executing within the executer), the executer 


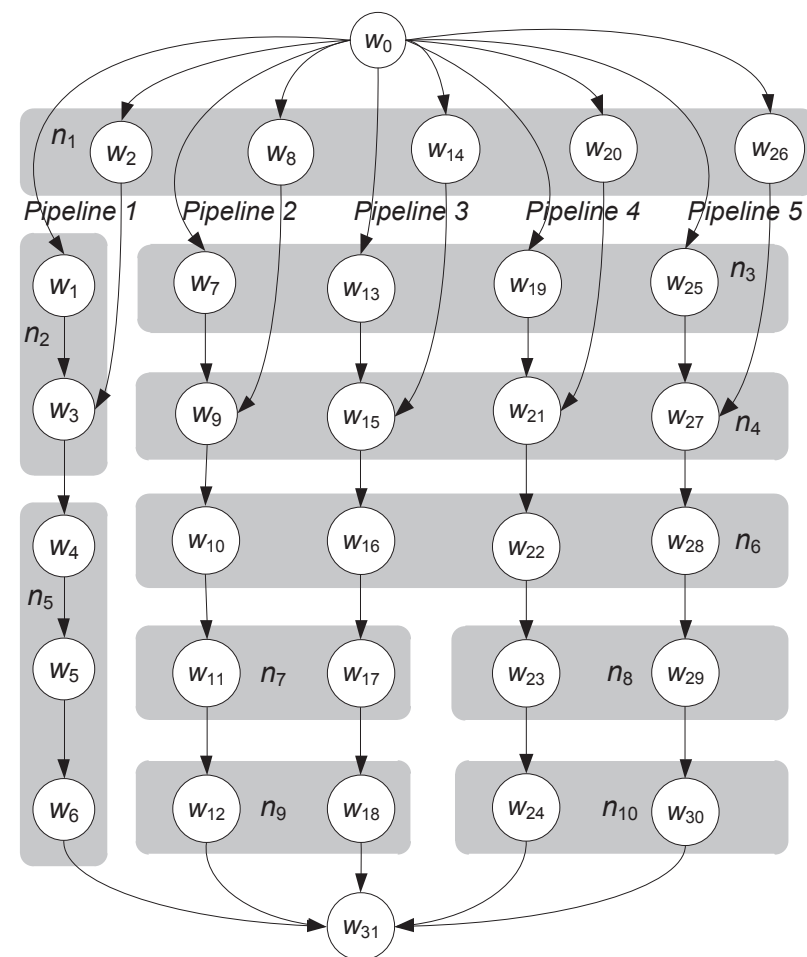

Figure 14: Composite climate modeling workflow structure mapped for execution.

employs an on-node scheduling policy, either FS or CPPS, to control the execution priorities of those concurrent modules.

4. Once a module finishes, the sender of the controller sends a finish message to each of its succeeding modules.

ClusterWE employs multi-threading to increase the performance measurement accuracy. Since each compute node in the PC cluster has 12 CPU cores, we set the CPU affinities of the controller and the executer to be any two most lightly loaded cores on the mapping node to minimize the negative effect of undesired resource competition and system dynamics for performance evaluation.

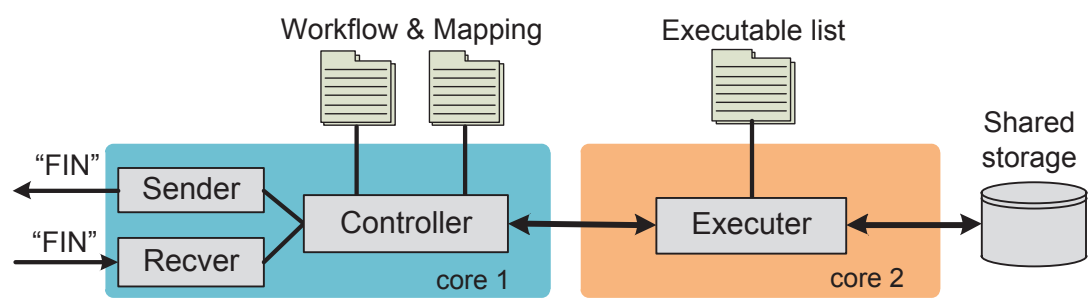

Figure 15: The architecture of a PC Cluster-based Workflow Engine. 


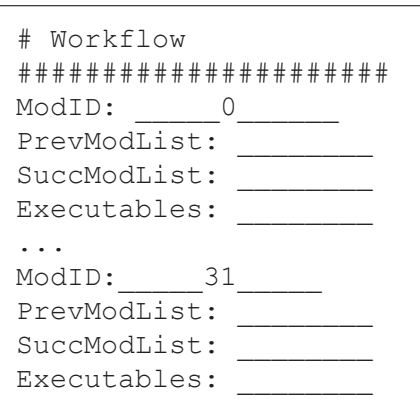

(a)

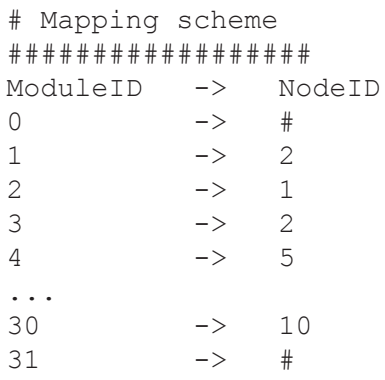

(b)

Figure 16: (a) workflow structure file; and (b) workflow mapping scheme file in ClusterWE.

\subsubsection{Experimental Results}

We run the workflow shown in Fig. 14 for 10 times under both FS as used by existing methods, and CPPS as used by MSI in the PC cluster, and calculate the average execution time of each workflow module, as tabulated in Table 4. The critical path of execution time under FS is: $w_{0} \rightarrow w_{2} \rightarrow w_{3} \rightarrow w_{4} \rightarrow w_{5} \rightarrow w_{6} \rightarrow w_{31}$, and the corresponding end-to-end delay is 1987.12 seconds; while under MSI, the critical path is: $w_{0} \rightarrow w_{20} \rightarrow$ $w_{21} \rightarrow w_{22} \rightarrow w_{23} \rightarrow w_{24} \rightarrow w_{31}$, and the corresponding end-to-end delay is 1498.02 seconds. Compared with FS scheduling under a given mapping scheme, MSI achieves an average $24.61 \%$ performance improvement in terms of end-to-end delay by invoking a more sophisticated CPPS on-node scheduling procedure.

Table 4: The average execution time (seconds) of the workflow modules under both FS and MSI.

\begin{tabular}{|c|c|l|l|c|c|c|c|}
\hline Mod & Node & \multicolumn{2}{|c|}{ Time (seconds) } & \multirow{2}{*}{ Mod } & Node & \multicolumn{2}{|c|}{ Time (seconds) } \\
\cline { 8 - 8 } & $n$ & FS & MSI & $w$ & $n$ & FS & MSI \\
\hline$w_{0}$ & - & 0.0 & 0.0 & $w_{16}$ & $n_{6}$ & 2.7156 & 2.4939 \\
\hline$w_{1}$ & $n_{2}$ & 3.8329 & 3.6956 & $w_{17}$ & $n_{7}$ & 158.89 & 163.57 \\
\hline$w_{2}$ & $n_{1}$ & 1355.9 & 618.73 & $w_{18}$ & $n_{9}$ & 1.5051 & 1.4471 \\
\hline$w_{3}$ & $n_{2}$ & 27.375 & 26.890 & $w_{19}$ & $n_{3}$ & 10.894 & 8.8737 \\
\hline$w_{4}$ & $n_{5}$ & 2.7217 & 3.0708 & $w_{20}$ & $n_{1}$ & 1169.5 & 1296.5 \\
\hline$w_{5}$ & $n_{5}$ & 599.51 & 600.01 & $w_{21}$ & $n_{4}$ & 16.580 & 15.566 \\
\hline$w_{6}$ & $n_{5}$ & 1.6512 & 1.6975 & $w_{22}$ & $n_{6}$ & 2.4337 & 3.0668 \\
\hline$w_{7}$ & $n_{3}$ & 10.923 & 10.161 & $w_{23}$ & $n_{8}$ & 189.59 & 181.35 \\
\hline$w_{8}$ & $n_{1}$ & 1164.3 & 1101.4 & $w_{24}$ & $n_{10}$ & 1.5401 & 1.5305 \\
\hline$w_{9}$ & $n_{4}$ & 16.572 & 15.094 & $w_{25}$ & $n_{3}$ & 10.829 & 10.880 \\
\hline$w_{10}$ & $n_{6}$ & 2.7402 & 2.4877 & $w_{26}$ & $n_{1}$ & 1351.8 & 1245.4 \\
\hline$w_{11}$ & $n_{7}$ & 159.30 & 164.42 & $w_{27}$ & $n_{4}$ & 17.371 & 15.659 \\
\hline$w_{12}$ & $n_{9}$ & 1.4488 & 1.4606 & $w_{28}$ & $n_{6}$ & 2.5658 & 2.5330 \\
\hline$w_{13}$ & $n_{3}$ & 10.796 & 9.0182 & $w_{29}$ & $n_{8}$ & 189.60 & 179.73 \\
\hline$w_{14}$ & $n_{1}$ & 1137.7 & 1213.5 & $w_{30}$ & $n_{10}$ & 1.5340 & 1.5242 \\
\hline$w_{15}$ & $n_{4}$ & 15.507 & 14.456 & $w_{31}$ & - & 0.0 & 0.0 \\
\hline
\end{tabular}




\section{Conclusion}

We proposed an integrated Mapping and Scheduling Interaction (MSI) algorithm that incorporates scheduling into mapping for workflow performance optimization. Extensive simulation and experimental results on real-life scientific workflows illustrated that MSI is able to improve the end-to-end performance by exploring the interactions between mapping and scheduling. It is of our future interest to perform this integration in a dynamic manner and test it on scientific workflows of larger scales in wide-area production networks. It is also of our future interest to explore finer-grained control mechanisms beyond system commands "nice" and "renice" to realize a higher precision in CPU allocation for further performance improvement of MSI.

\section{Acknowledgments}

This research is sponsored by U.S. Department of Energy's Office of Science under Grant No. DE-SC0010641 with University of Memphis.

\section{References}

[1] The Office of Science Data-Management Challenge. Report from the DOE Office of Science Data-Management Workshops, Technical Report SLAC-R-782, SLAC, Available online at: http://www.slac.stanford.edu/cgi-wrap/getdoc/slac-r-782.pdf (March-May 2004).

[2] G. Juve, A. Chervenak, E. Deelman, S. Bharathi, G. Mehta, K. Vahi, Characterizing and Profiling Scientific Workflows, Future Generation Computer Systems 29 (3) (2013) 682-692.

[3] HTCondor/DAGMan, http://research.cs.wisc.edu/htcondor/.

[4] J. Frey, T. Tannenbaum, M. Livny, I. Foster, S. Tuecke, Condor-G: A Computation Management Agent for Multi-Institutional Grids, Cluster Computing 5 (3) (2002) 237-246.

[5] E. Deelman, S. Callaghan, E. Field, H. Francoeur, R. Graves, N. Gupta, V. Gupta, T. Jordan, C. Kesselman, P. Maechling, J. Mehringer, G. Mehta, D. Okaya, K. Vahi, L. Zhao, Managing Large-Scale Workflow Execution from Resource Provisioning to Provenance Tracking: The CyberShake Example, in: Proc. of the 2nd Int'l Conference on e-Science and Grid Computing, Amsterdam, Netherlands, 2006, Article 14.

[6] B. Ludäscher, I. Altintas, C. Berkley, D. Higgins, E. Jaeger-Frank, M. Jones, E. Lee, J. Tao, Y. Zhao, Scientific Workflow Management and the Kepler System, Concurrency and Computation: Practice and Experience 18 (10) (2006) 1039-1605.

[7] Pegasus Workflow Management System, https://pegasus.isi.edu/. 
[8] E. Deelman, J. Blythe, Y. Gil, C. Kesselman, G. Mehta, S. Patil, M. Su, K. Vahi, M. Livny, Pegasus: Mapping Scientific Workflows onto the Grid, in: Proc. of the 2nd European AcrossGrids Conference, Nicosia, Cyprus, 2004, pp. 11-20.

[9] D. Churches, G. Gombas, A. Harrison, J. Maassen, C. Robinson, M. Shields, I. Taylor, I. Wang, Programming Scientific and Distributed Workflow with Triana Services, Concurrency and Computation: Practice and Experience, Special Issue on Workflow in Grid Systems 18 (10) (2006) 1021-1037.

[10] T. Fahringer, A. Jugravu, S. Pllana, R. Prodan, J. C. Seragiotto, H.-L. Truong, ASKALON: A Tool Set for Cluster and Grid Computing, Concurrency and Computation: Practice and Experience 17 (2-4) (2005) 143-169.

[11] B. Wassermann, W. Emmerich, B. Butchart, N. Cameron, L. Chen, J. Patel, Workflows for e-Science: Scientific Workflows for Grids, Springer, London, 2007, Ch. Sedna: a BPEL-Based Environment for Visual Scientific Workflow Modeling, pp. 427-448.

[12] D. Fernández-Baca, Allocating Modules to Processors in a Distributed System, IEEE Transactions on Software Engineering 15 (11) (1989) 1427-1436.

[13] Q. Wu, D. Yun, X. Lin, Y. Gu, W. Lin, Y. Liu, On Workflow Scheduling for End-to-end Performance Optimization in Distributed Network Environments, in: Proc. of the 16th Workshop on Job Scheduling Strategies for Parallel Processing, Shanghai, China, 2012, pp. 76-95.

[14] CGroups, http://www.kernel.org/doc/Documentation/cgroups/cgroups.txt.

[15] OSCARS: On-demand Secure Circuits and Advance Reservation System, http://www.es.net/oscars .

[16] Internet2 Interoperable On-Demand Network (ION) Service, http://www . internet2. edu/ion.

[17] S. Blake, D. Black, M. Carlson, E. Davies, Z. Wang, W. Weiss, An Architecture for Differentiated Services, IETF RFC 2475 (December 1998).

[18] R. Braden, D. Clark, S. Shenker, Integrated Services in the Internet Architecture: an Overview, IETF RFC 1683 (June 1994).

[19] R. Braden, L. Zhang, S. Berson, S. Herzog, S. Jamin, Resource ReSerVation Protocol (RSVP), http://tools.ietf.org/rfc/rfc2205.txt (Sep. 1997).

[20] L. Andersson, G. Swallow, The Multiprotocol Label Switching (MPLS) Working Group decision on MPLS signaling protocols, IETF RFC 3468 (Feb. 2003).

[21] E. Deelman, D. Gannon, M. Shields, I. Taylor, Workflows and e-Science: An Overview of Workflow System Features and Capabilities, Future Generation Computer Systems 25 (5) (2009) 528-540. 
[22] D. Yun, Q. Wu, Y. Gu, X. Liu, On an Integrated Mapping and Scheduling Solution to Largescale Scientific Workflows in Resource Sharing Environments, in: Proc. of the 46th Annual Simulation Symposium, San Diego, CA, USA, 2013, Article 7, 8 pages.

[23] W. Chen, R. Silva, E. Deelman, R. Sakellariou, Balanced Task Clustering in Scientific Workflows, in: Proc. of the 9th IEEE Int'l Conference on e-Science, Beijing, China, 2013, pp. $188-195$.

[24] J. Cao, S. Jarvis, S. Saini, G. Nudd, GridFlow: Workflow Management for Grid Computing, in: Proc. of the 3st IEEE/ACM Intl. Symposium on Cluster Computing and the Grid, Tokyo, Japan, 2003, pp. 198-205.

[25] T. Ma, R. Buyya, Critical-Path and Priority based Algorithms for Scheduling Workflows with Parameter Sweep Tasks on Global Grids, in: Proc. of the 17th Intl. Symposium on Computer Architecture and High Performance Computing, Rio de Janeiro, Brazil, 2005, pp. 251-258.

[26] L. Chen, G. Agrawal, Resource Allocation in a Middleware for Streaming Data, in: Proc. of the 2nd Workshop on Middleware for Grid Computing, Toronto, Ontario, Canada, 2004, pp. $5-10$.

[27] V. Chaudhary, J. Aggarwal, A Generalized Scheme for Mapping Parallel Algorithms, IEEE Transactions on Parallel and Distributed Systems 4 (3) (1993) 328-346.

[28] H. Topcuoglu, S. Hariri, M. Wu, Performance-Effective and Low-Complexity Task Scheduling for Heterogeneous Computing, IEEE Transactions on Parallel and Distributed Systems 13 (3) (2002) 260-274.

[29] G. Malewicz, I. Foster, A. Rosenberg, M. Wilde, A Tool for Prioritizing DAGMan Jobs and Its Evaluation, in: Proc. of the 15th IEEE Int'l Symposium on High Performance Distributed Computing, Paris, France, 2006, pp. 156-168.

[30] S. Ranaweera, D. Agrawal, A Task Duplication Based Scheduling Algorithm for Heterogeneous Systems, in: Proc. of the 14th Int'l Parallel and Distributed Processing Symposium, Cancun, Mexico, 2000, pp. 445-450.

[31] R. Bajaj, D. Agrawal, Improving Scheduling of Tasks in a Heterogeneous Environment, IEEE Transactions on Parallel and Distributed Systems 15 (2) (2004) 107-118.

[32] T. Braun, H. Siegel, N. Beck, L. Boloni, M. Maheswaran, A. Reuther, J. Robertson, M. Theys, B. Yao, D. Hensgen, R. Freund, A Comparison of Eleven Static Heuristics for Mapping a Class of Independent Tasks onto Heterogeneous Distributed Computing Systems, Journal of Parallel and Distributed Computing 61 (6) (2001) 810-837. 
[33] M. Maheswaran, H. Siegel, A Dynamic Matching and Scheduling Algorithm for Heterogeneous Computing Systems, in: Proc. of the 7th Heterogeneous Computing Workshop, Washington, DC, USA, 1998, pp. 57-69.

[34] B. Messmer, Efficient Graph Matching Algorithms for Preprocessed Model Graphs, Ph.D. thesis, Institute of Computer Science and Applied Mathematics, University of Bern (1996).

[35] L. Cordella, P. Foggia, C. Sansone, M. Vento, An improved algorithm for matching large graphs, in: Proc. of the 3rd IAPR-TC15 Workshop on Graph-based Representations in Pattern Recognition, Ischia, Italy, 2001, pp. 149-159.

[36] Y. Gu, Q. Wu, N. Rao, Analyzing Execution Dynamics of Scientific Workflows for Latency Minimization in Resource Sharing Environments, in: Proc. of the 7th IEEE World Congress on Services, Washington, DC, USA, 2011, pp. 153-160.

[37] Y. Kwok, I. Ahmad, Dynamic Critical-Path Scheduling: An Effective Technique for Allocating Task Graphs to Multiprocessors, IEEE Transactions on Parallel and Distributed Systems 7 (5) (1996) 506-521.

[38] C. Boeres, J. Filho, V. Rebello, A Cluster-based Strategy for Scheduling Task on Heterogeneous Processors, in: Proc. of the 16th Symposium on Computer Architecture and High Performance Computing, Washington, DC, USA, 2004, pp. 214-221.

[39] A. Gerasoulis, T. Yang, A Comparison of Clustering Heuristics for Scheduling Directed Acyclic Graphs on Multiprocessors, Journal of Parallel and Distributed Computing 16 (4) (1992) 276291.

[40] I. Ahmed, Y. Kwok, On Exploiting Task Duplication in Parallel Program Scheduling, IEEE Transactions on Parallel and Distributed Systems 9 (9) (1998) 872-892.

[41] M. Dhodhi, I. Ahmad, A. Yatama, An Integrated Technique for Task Matching and Scheduling onto Distributed Heterogeneous Computing Systems, Journal of Parallel and Distributed Computing 62 (9) (2002) 1338-1361.

[42] E. Hou, N. Ansari, H. Ren, A Genetic Algorithm for Multiprocessor Scheduling, IEEE Transactions on Parallel and Distributed Systems 5 (2) (1994) 113-120.

[43] A. Wu, H. Yu, S. Jin, K.-C. Lin, G. Schiavone, An Incremental Genetic Algorithm Approach to Multiprocessor Scheduling, IEEE Transactions on Parallel and Distributed Systems 15 (9) (2004) 824-834.

[44] A. Benoit, Y. Robert, Mapping Pipeline Skeletons onto Heterogeneous Platforms, Journal of Parallel and Distributed Computing 68 (6) (2008) 790-808. 
[45] M. Garey, D. Johnson, Computers and Intractability: A Guide to the Theory of NPcompleteness, W. H. Freeman, San Francisco, 1979.

[46] D. Goyal, Scheduling Processor Bound Systems, Tech. Rep. CS-76-036, Computer Science Department, Washington State University, Pullman, WA, USA (Nov. 1976).

[47] Q. Wu, Y. Gu, M. Zhu, N. Rao, Optimizing Network Performance of Computing Pipelines in Distributed Environments, in: Proc. of the 22nd IEEE Int'l Parallel and Distributed Processing Symposium, Miami, Florida, 2008, pp. 1-8.

[48] Q. Wu, Y. Gu, Supporting Distributed Application Workflows in Heterogeneous Computing Environments, in: Proc. of the 14th IEEE Int'l Conference on Parallel and Distributed Systems, Melbourne, Australia, 2008, pp. 3-10.

[49] Q. Wu, Y. Gu, Optimizing end-to-end performance of data-intensive computing pipelines in heterogeneous network environments, Journal of Parallel and Distributed Computing 71 (2) (2011) 254-265.

[50] B. Manoj, A. Sekhar, C. Murthy, A State-space Search Approach for Optimizing Reliability and Cost of Execution in Distributed Sensor Networks, Journal of Parallel and Distributed Computing 69 (1) (2009) 12-19.

[51] The Weather Research \& Forecasting Model, http://www.wrf-model.org/index.php.

[52] Biological, Environmental \& Climate Sciences Department, Brookhaven National Laboratory, http://www.bnl.gov/ebnn/becs/.

[53] Q. Wu, V. Datla, On Performance Modeling and Prediction in Support of Scientific Workflow Optimization, in: Proc. of the 2011 IEEE World Congress on Services, Washington, DC, 2011, pp. $161-168$. 